\title{
Operators in pre-Riesz spaces: moduli and homomorphisms
}

\author{
Anke Kalauch $^{1} \cdot$ Janko Stennder ${ }^{1}\left[\right.$. Onno van Gaans ${ }^{2}$ \\ Received: 25 September 2020 / Accepted: 27 July 2021 / Published online: 30 August 2021 \\ (c) The Author(s) 2021
}

\begin{abstract}
We focus on two topics that are related to moduli of elements in partially ordered vector spaces. First, we relate operators that preserve moduli to generalized notions of lattice homomorphisms, such as Riesz homomorphisms, Riesz* homomorphisms, and positive disjointness preserving operators. We also consider complete Riesz homomorphisms, which generalize order continuous lattice homomorphisms. Second, we characterize elements with a modulus by means of disjoint elements and apply this result to obtain moduli of functionals and operators in various settings. On spaces of continuous functions, we identify those differences of Riesz* homomorphisms that have a modulus. Many of our results for pre-Riesz spaces of continuous functions lead to results on order unit spaces, where the functional representation is used.
\end{abstract}

Keywords Complete Riesz homomorphism · Disjoint elements · Disjointness preserving operator · Functional representation - Modulus · Order unit space · Partially ordered vector space $\cdot$ Pre-Riesz space $\cdot$ Riesz homomorphism

Mathematics Subject Classification 06F20 - 47B60

\section{Introduction}

One of the important features of the lattice structure in a vector lattice is that every element $x$ has a modulus $|x|$. In a partially ordered vector space that is not a lattice, some elements do have a modulus and some do not. As a first theme in this paper,

Janko Stennder

Janko.Stennder@tu-dresden.de

Anke Kalauch

anke.kalauch@tu-dresden.de

Onno van Gaans

vangaans@math.leidenuniv.nl

1 Fakultät Mathematik, Institut für Analysis, TU Dresden, 01062 Dresden, Germany

2 Mathematical Institute, Leiden University, P.O. Box 9512, 2300, RA Leiden, The Netherlands 
we study operators that preserve moduli, and related notions of homomorphisms. The second theme is to characterize the elements in a pre-Riesz space that have a modulus.

In vector lattices, Riesz homomorphisms are the linear operators that preserve moduli. Various generalizations to partially ordered vector spaces have been studied, such as Riesz* homomorphisms [10] and Riesz homomorphisms [5]. Complete Riesz homomorphisms [5] generalize the notion of order continuous Riesz homomorphisms on vector lattices. These notions of homomorphisms in partially ordered vector spaces are defined by means of relations of sets of upper bounds. Furthermore, one considers operators that preserve moduli or disjointness. We establish new properties and relations between these classes of homomorphisms.

Concerning the second theme, it is difficult, in general, to determine which elements have a modulus, in particular in spaces of operators. For operators between vector lattices, the modulus of an operator is known to be given by the Riesz-Kantorovich formula only in specific cases. We characterize elements with a modulus in pre-Riesz spaces. Hereby, we use vector lattice covers of pre-Riesz spaces. In particular, we consider moduli of functionals and certain instances of operators on order unit spaces.

A large part of the paper deals with pre-Riesz spaces of continuous functions. These results can be applied to order unit spaces by means of their functional representations. More precisely, we consider an order unit space $(X, K, u)$ and its functional representation $\Phi: X \rightarrow \mathrm{C}(\bar{\Lambda})$, where $\Lambda$ consists of the extreme points of the set of positive linear functionals $\varphi: X \rightarrow \mathbb{R}$ with $\varphi(u)=1$, and where we consider the weak-* topology on the dual of $X$. Hayes [11, Corollary 1] has established that the elements of $\Lambda$ are exactly the nonzero Riesz homomorphisms on $X$ scaled to be one at $u$, and it is due to van Haandel [10, Theorem 5.10(iii)] that $\bar{\Lambda}$ consists of the scaled nonzero Riesz* homomorphisms. So far, it has been unknown which elements of $\Lambda$ are complete Riesz homomorphisms.

The first theme (homomorphisms) is investigated in the Sects. 3-6 and 8, whereas the second theme (existence of moduli) is considered in the Sects. 7 and 9. Note that the discussions of the two themes are not strictly separated, as the themes are interrelated. The necessary preliminaries are listed in Sect. 2. In Sect. 3, we prove some new properties of the van Haandel extension of Riesz* homomorphisms, and Sect. 4 is devoted to basic properties of Riesz* homomorphisms and complete Riesz homomorphisms on order unit spaces. In Sect. 5, we show that the scaled nonzero complete Riesz homomorphisms from $X$ to $\mathbb{R}$ are the $\varphi \in \bar{\Lambda}$ for which $\{\varphi\}$ is open in $\bar{\Lambda}$, see Proposition 24 below. On pre-Riesz spaces, every complete Riesz homomorphism is an order continuous Riesz homomorphism [9, Proposition 2.3.20]. Example 26 below shows that the converse is not true even for functionals.

Our results on relations of homomorphisms (Sects. 6 and 8) are summarized in the following scheme. 


\begin{tabular}{|c|c|c|}
\hline $\begin{array}{c}\text { o-continuous Riesz homomorphism } \\
\Downarrow \Uparrow\end{array}$ & $\Rightarrow(\nLeftarrow)$ & $\begin{array}{c}\text { Riesz homomorphism } \\
\Downarrow \Uparrow(\Lambda=\bar{\Lambda})\end{array}$ \\
\hline complete Riesz homomorphism & $(\bar{\Lambda}$ discrete $)$ & $\begin{array}{c}\text { Riesz* homomorphism } \\
\Downarrow \Uparrow \text { (SD) } \\
\text { positive disjointness } \\
\text { preserving operator }\end{array}$ \\
\hline
\end{tabular}

In Sect. 6, we show that if $\bar{\Lambda}=\Lambda$, that is, every Riesz* homomorphism from $X$ to $\mathbb{R}$ is a Riesz homomorphism, then every Riesz* homomorphism from $X$ to $Y$ is a Riesz homomorphism, where $Y$ is an arbitrary order unit space. Similarly, if $\bar{\Lambda}$ is discrete, i.e., every Riesz* homomorphism from $X$ into $\mathbb{R}$ is a complete Riesz homomorphism, then every Riesz* homomorphism from $X$ to $Y$ is a complete Riesz homomorphism, see Theorem 29 below.

In Riesz spaces, an operator is a Riesz homomorphism if and only if it is a positive disjointness preserving operator. In pre-Riesz spaces, every Riesz* homomorphism is positive and disjointness preserving [9, Theorem 5.1.12], and the converse is not true, in general [13, Example 2.29]. In Sect. 8, we provide sufficient conditions on the domain such that every positive disjointness preserving operator is a Riesz* homomorphism. More precisely, if $P$ is a nonempty compact Hausdorff space and $X$ is an order dense subspace of $\mathrm{C}(P)$ such that the points of $P$ are separated by disjoint elements of $X$ (condition (SD)), we show that every positive disjointness preserving operator from $X$ into an appropriate range space is a Riesz* homomorphism, see Theorem 49 below. A similar result for order unit spaces follows in Corollary 51.

Concerning the second theme, in Sect. 7, we show that an element $x$ of a pre-Riesz space $X$ has a modulus if and only if $|i(x)| \in i[X]$, where $i: X \rightarrow Y$ embeds into a vector lattice cover $Y$ of $X$, see Theorem 35(ii) below. We also relate the modulus to disjointness and show that the existence of $|x|$ is equivalent to the existence of two positive disjoint elements $a$ and $b$ in $X$ with $x=a-b$, see Lemma 34. Consequently, an operator preserves moduli if and only if it is disjointness preserving on the positive elements, see Proposition 39. In Sect. 9, we apply these results to functionals and operators. For some specific cases, we calculate the set of operators with modulus. For instance, we identify the differences of Riesz* homomorphisms on certain preRiesz spaces of continuous functions that have a modulus, see Proposition 60 and Theorem 63. This leads to a discussion on the disjointness of Riesz* homomorphisms in operator spaces.

\section{Preliminaries}

Let $X$ be a real vector space containing a cone $K$, i.e., $K$ is convex, $\lambda K \subseteq K$ for every $\lambda \geq 0$, and $K \cap(-K)=\{0\}$. The cone $K$ induces a partial order $\leq$ in $X$ by $x \leq y$ if $y-x \in K$. We call $(X, K)$ a partially ordered vector space. We mostly assume that $(X, K)$ is directed, meaning that $X=K-K$. The space $(X, K)$ is called Archimedean if, for every $x, y \in X$ with $n x \leq y$ for all $n \in \mathbb{N}$, we have $x \leq 0$. A 
linear subspace $D$ of $X$ is order dense in $X$ if, for every $x \in X$, we have

$$
x=\inf \{d \in D ; d \geq x\} .
$$

The concept of order denseness originated in [5, p. 360]. A linear map $i: X \rightarrow Y$, where $X$ and $Y$ are partially ordered vector spaces, is called bipositive if for every $x \in X, x \geq 0$ is equivalent to $i(x) \geq 0$.

A partially ordered vector space $X$ is called a pre-Riesz space if there is a Riesz space $Y$ and a bipositive linear map $i: X \rightarrow Y$ such that $i[X]$ is order dense in $Y$. We call $(Y, i)$ a vector lattice cover of $X$. An intrinsic definition of pre-Riesz spaces is given by van Haandel in [10], see also [9, Section 2.2]. Note that every directed Archimedean partially ordered vector space is pre-Riesz, and that every pre-Riesz space is directed, see, e.g., [9, Proposition 2.2.3]. Clearly, every Riesz space is preRiesz. For standard notations in Riesz spaces, see [2]. If $(Y, i)$ is a vector lattice cover of a pre-Riesz space $X$ such that no proper Riesz subspace of $Y$ contains $i[X]$, then we call $(Y, i)$ a Riesz completion of $X$. Such a space is unique up to isomorphism (for details see, e.g., [9, Section 2.4]). Given a concrete example of a partially ordered vector space, the challenge is to find a convenient realization of a vector lattice cover such that the order in the vector lattice cover is pointwise. In general this is hard, but one can use the functional representation in case of order unit spaces. Also for some spaces of operators, it is possible to calculate such a vector lattice cover, see Example 59.

A pre-Riesz space $X$ with Riesz completion $(Y, i)$ is called pervasive if for every $y \in Y \backslash\{0\}, y \geq 0$, there is $x \in X \backslash\{0\}$ such that $0 \leq i(x) \leq y$. Instead of the Riesz completion, one can also use an arbitrary vector lattice cover in this definition, see [9, Proposition 2.8.8].

For $A \subseteq X$, denote $A^{\mathrm{u}}=\{x \in X ; \forall a \in A: x \geq a\}$ and $A^{1}=\{x \in X ; \forall a \in$ $A: x \leq a\}$. Riesz* homomorphisms are defined in [10, Definition 5.1 and Corollary 5.4(iv)], Riesz homomorphisms and complete Riesz homomorphisms in [5].

Definition 1 Let $X$ and $Y$ be directed partially ordered vector spaces. A linear map $T: X \rightarrow Y$ is called

- a Riesz* homomorphism if, for every nonempty finite subset $F$ of $X$, one has

$$
T\left[F^{\mathrm{ul}}\right] \subseteq T[F]^{\mathrm{ul}}
$$

- a Riesz homomorphism if, for every $x, y \in X$, one has

$$
T\left[\{x, y\}^{\mathrm{u}}\right]^{1}=T[\{x, y\}]^{\mathrm{ul}},
$$

- a complete Riesz homomorphism if, for every nonempty set $A \subseteq X$, we have

$$
\inf A=0 \Longrightarrow \inf T[A]=0 \text {. }
$$

If $X$ and $Y$ are pre-Riesz spaces, then every complete Riesz homomorphism is a Riesz homomorphism, every Riesz homomorphism is a Riesz* homomorphism, and every 
Riesz* homomorphism is positive, see [9, Theorem 2.3.19]. If $X$ is, in addition, a vector lattice, then $T$ is a Riesz homomorphism if and only if, for every $u, v \in X$, there exists $T(u) \vee T(v)$ in $Y$, and $T(u) \vee T(v)=T(u \vee v)$. If $X$ and $Y$ are vector lattices, then the notions of a Riesz homomorphism and a Riesz* homomorphism both coincide with the notion of a Riesz homomorphism from vector lattice theory, see, e.g., [9, Lemma 2.3.2]. Moreover, in this case, $T$ is a complete Riesz homomorphism if and only if $T$ is an order continuous Riesz homomorphism, see [9, Proposition 1.4.5].

We will need the following characterization of Riesz* homomorphisms and Riesz homomorphisms in the case that the range space is a vector lattice, see also [9, Proposition 2.3.26].

Proposition 2 Let $X$ be a partially ordered vector space, let $Y$ be a Riesz space, and let $T: X \rightarrow Y$ be a linear map.

(i) $T$ is a Riesz* homomorphism if and only if

$$
\sup \left\{T(x) ; x \in\left\{a_{1}, \ldots, a_{n}\right\}^{\mathrm{ul}}\right\}=\bigvee_{k=1}^{n} T\left(a_{k}\right)
$$

for every $a_{1}, \ldots, a_{n} \in X$ and $n \in \mathbb{N}$.

(ii) $T$ is a Riesz homomorphism if and only if

$$
\inf \left\{T(x) ; x \in\left\{a_{1}, \ldots, a_{n}\right\}^{\mathrm{u}}\right\}=\bigvee_{k=1}^{n} T\left(a_{k}\right)
$$

for every $a_{1}, \ldots, a_{n} \in X$ and $n \in \mathbb{N}$.

The following modification of Proposition 2(ii) will be of use.

Proposition 3 Let $X_{1}$ be a partially ordered vector space, $Y$ a Riesz space, $X_{2}$ an order dense subspace of $Y$, and $T: X_{1} \rightarrow X_{2}$ a linear map. $T$ is a Riesz homomorphism if and only if, for every $x, y \in X_{1}$, we have

$$
\inf \left\{T v ; v \in X_{1}, v \in\{x, y\}^{\mathrm{u}}\right\}=T(x) \vee T(y) \text { in } Y .
$$

Proof Let $T: X_{1} \rightarrow X_{2}$ be a Riesz homomorphism. Let $x, y \in X_{1}$. Since $T$ is positive, $T x \vee T y$ is a lower bound of $\left\{T v ; v \in X_{1}, v \in\{x, y\}^{\mathrm{u}}\right\}$. Let $w \in Y$ be a lower bound of $\left\{T v ; v \in X_{1}, v \in\{x, y\}^{\mathrm{u}}\right\}$. As $X_{2}$ is order dense in $Y$, it suffices to show that, for every $z \in X_{2}$ with $z \leq w$, we have that $z \leq T x \vee T y$. Let $z \in X_{2}$ be such that $z \leq w$. Then we have

$$
z \in\left\{T v ; v \in X_{1}, v \in\{x, y\}^{\mathrm{u}}\right\}^{1}=\{T x, T y\}^{\mathrm{ul}}
$$

in $X_{2}$, as $T$ is a Riesz homomorphism. Hence $z \leq T x \vee T y$ in $Y$, which implies $w \leq T x \vee T y$.

Conversely, let $x, y \in X_{1}$. We have that

$$
\left\{T v ; v \in X_{1}, v \in\{x, y\}^{\mathrm{u}}\right\}^{1}=\{T x \vee T y\}^{1}=\{T x, T y\}^{\mathrm{ul}}
$$


in $Y$. Intersecting with $X_{2}$ yields that $T$ is a Riesz homomorphism.

Recall that two elements $x$ and $y$ in a pre-Riesz space $(X, K)$ are disjoint, denoted $x \perp y$, if $\{x+y, x-y\}^{\mathrm{u}}=\{x-y,-x+y\}^{\mathrm{u}}$. The disjoint complement of a set $M \subseteq X$ is denoted by $M^{\mathrm{d}}$. If $(Y, i)$ is a vector lattice cover of $X$, then $x \perp y$ if and only if $i(x) \perp i(y)$, see, e.g., [9, Proposition 4.1.4]. Let $X$ and $V$ be pre-Riesz spaces. A linear map $T: X \rightarrow V$ is called disjointness preserving if, for every $x, y \in X$ with $x \perp y$, one has $T(x) \perp T(y)$. If $T$ is a Riesz* homomorphism, then $T$ is a positive disjointness preserving operator, see [9, Theorem 5.1.12].

We collect standard definitions concerning operators. Let $X, Y$ be a partially ordered vector spaces. As usual, a linear operator $T$ from $X$ to $Y$ is called positive if $T$ maps the cone in $X$ into the cone in $Y$, order bounded if $T$ maps order bounded subsets to order bounded subsets, and regular whenever $T$ can be written as a difference of two positive operators. For the set of all linear operators, we write $\mathrm{L}(X, Y)$. The set of all order bounded operators is denoted by $\mathrm{L}^{\mathrm{b}}(X, Y)$, whereas the set of all regular operators is denoted by $\mathrm{L}^{\mathrm{r}}(X, Y)$. If $X$ is directed, the set of all positive operators is a cone, which yields a natural partial order in these spaces of operators. Recall that $\mathrm{L}^{\mathrm{r}}(X, Y) \subseteq \mathrm{L}^{\mathrm{b}}(X, Y)$. If $X$ is directed and has the Riesz decomposition property, and $Y$ is a Dedekind complete vector lattice, then $\mathrm{L}^{\mathrm{b}}(X, Y)=\mathrm{L}^{\mathrm{r}}(X, Y)$ is a Dedekind complete vector lattice, and the lattice operations are given by the Riesz-Kantorovich formulas, see, e.g., [3, Theorem 1.59].

We say that a net $\left(x_{\alpha}\right)_{\alpha \in A}$ in a partially ordered vector space $X$ order converges to $x \in X$ if there is a net $\left(y_{\alpha}\right)_{\alpha \in A}$ with $y_{\alpha} \downarrow 0$ (which means that the net is decreasing and $\inf \left\{y_{\alpha} ; \alpha \in A\right\}=0$ ) and $\alpha_{0} \in A$ such that, for every $\alpha \in A, \alpha \geq \alpha_{0}$, we have $\pm\left(x_{\alpha}-x\right) \leq y_{\alpha}$. In this case, we write $x_{\alpha} \stackrel{o}{\rightarrow} x$. The following statement on order continuity in finite dimensions is convenient if one studies examples. Note that the (Euclidean) norm in $\mathbb{R}^{n}$ is semimonotone if the cone $K$ in $\mathbb{R}^{n}$ is generating and closed, for details, see, e.g., [9, Proposition 1.5.16]. Therefore, order bounded sets are norm bounded, see [9, Lemma 1.5.7]. In particular, every order interval $[a, b]:=\{x \in$ $\left.\mathbb{R}^{n} ; a \leq x \leq b\right\}$ in $(\mathbb{R}, K)$ is compact.

Proposition 4 Let $K$ be a generating closed cone in $\mathbb{R}^{n}$.

(i) Let $\left(x_{\alpha}\right)_{\alpha \in A}$ be a net in $\mathbb{R}^{n}$ and let $x \in \mathbb{R}^{n}$. If $x_{\alpha} \stackrel{o}{\rightarrow} x$, then $x_{\alpha} \stackrel{\|\cdot\|}{\rightarrow} x$.

(ii) Every continuous linear map $\varphi: \mathbb{R}^{n} \rightarrow \mathbb{R}$ is order continuous.

Proof (i) First, let $\left(x_{\alpha}\right)_{\alpha \in A}$ be a net in $\mathbb{R}^{n}$ such that $x_{\alpha} \downarrow 0$. We show that every subnet of $\left(x_{\alpha}\right)$ has a subnet converging to 0 , which implies $x_{\alpha} \stackrel{\|\cdot\|}{\longrightarrow} 0$, by [15, Proposition 2.1.31]. Indeed, let $\left(y_{\beta}\right)_{\beta \in B}$ be a subnet of $\left(x_{\alpha}\right)$ and let $g: B \rightarrow A$ be increasing with $y_{\beta}=x_{g(\beta)}$ for every $\beta \in B$ and $g[B]$ cofinal in $A$. Fix $\beta_{0} \in B$. For every $\beta \geq \beta_{0}$, we have $0 \leq y_{\beta} \leq y_{\beta_{0}}$, hence $y_{\beta}$ belongs to the order interval $\left[0, y_{\beta_{0}}\right]$, which is compact. Hence, there exists a subnet $\left(z_{\gamma}\right)_{\gamma \in C}$ of $\left(y_{\beta}\right)$ and $z \in\left[0, y_{\beta_{0}}\right]$ such that $\left\|z_{\gamma}-z\right\| \rightarrow 0$, see [15, Proposition 2.1.37]. Let $h: C \rightarrow B$ be increasing with $z_{\gamma}=y_{h(\gamma)}$ for every $\gamma \in C$ and $h[C]$ cofinal in $B$. To show that $z=0$, fix $\alpha \in A$. As $g[B]$ and $h[C]$ are cofinal, there is $\beta_{\alpha} \in B$ with $g\left(\beta_{\alpha}\right) \geq \alpha$ and $\gamma_{\alpha} \in C$ with $h\left(\gamma_{\alpha}\right) \geq \beta_{\alpha}$. For every $\gamma \geq \gamma_{\alpha}$, we have

$$
z_{\gamma} \leq z_{\gamma_{\alpha}}=y_{h\left(\gamma_{\alpha}\right)} \leq y_{\beta_{\alpha}}=x_{g\left(\beta_{\alpha}\right)} \leq x_{\alpha} .
$$


Since $K$ is closed, it follows that $z \leq x_{\alpha}$. From $x_{\alpha} \downarrow 0$, we obtain $z=0$.

Second, let $\left(x_{\alpha}\right)_{\alpha \in A}$ be a net in $\mathbb{R}^{n}$ and let $x \in \mathbb{R}^{n}$ be such that $x_{\alpha} \stackrel{o}{\rightarrow} x$. Then there is a net $\left(y_{\alpha}\right)_{\alpha \in A}$ with $y_{\alpha} \downarrow 0$ and $\alpha_{0} \in A$ such that, for every $\alpha \geq \alpha_{0}$, we have $-y_{\alpha} \leq x_{\alpha}-x \leq y_{\alpha}$. Due to the first part of the proof, we obtain $\left\|y_{\alpha}\right\| \rightarrow 0$. Since the norm is semimonotone, it follows that $\left\|x_{\alpha}-x\right\| \rightarrow 0$, see also [9, Theorems 3.6.3 and 3.6.8].

(ii) is an immediate consequence of (i).

\section{The van Haandel extension revisited}

Van Haandel observed that Riesz* homomorphisms are exactly those operators between pre-Riesz spaces that can be extended to Riesz homomorphisms between the corresponding Riesz completions, see [10, Theorem 5.6] or [9, Theorem 2.4.11].

Theorem 5 Let $X_{1}$ and $X_{2}$ be pre-Riesz spaces with Riesz completions $\left(Y_{1}, i_{1}\right)$ and $\left(Y_{2}, i_{2}\right)$, respectively. Let $T: X_{1} \rightarrow X_{2}$ be a linear map. The following statements are equivalent.

(i) T is a Riesz* homomorphism.

(ii) There exists a Riesz homomorphism $S: Y_{1} \rightarrow Y_{2}$ satisfying $S \circ i_{1}=i_{2} \circ T$.

Moreover, if (i) is satisfied, then the Riesz homomorphism in (ii) is unique.

The operator $S$ in Theorem 5(ii) is called the van Haandel extension of $T$. If the operator $T$ is a Riesz homomorphism or a complete Riesz homomorphism, somewhat stronger conclusions than those of Theorem 5 can be drawn. For Riesz homomorphisms, a stronger uniqueness result holds.

Proposition 6 Let $V$ and $W$ be Riesz spaces and let $X$ be an order dense subspace of $V$ that generates $V$ as a Riesz space. Let $T: V \rightarrow W$ be a positive linear operator. If $\left.T\right|_{X}: X \rightarrow W$ is a Riesz homomorphism, then $T$ is a Riesz homomorphism.

Proof First, let $x_{1}, \ldots, x_{n} \in X$. We show that

$$
T\left(x_{1} \vee \cdots \vee x_{n}\right)=T\left(x_{1}\right) \vee \cdots \vee T\left(x_{n}\right) .
$$

Indeed, as $T$ is positive, we have $T\left(x_{1} \vee \cdots \vee x_{n}\right) \geq T\left(x_{1}\right) \vee \cdots \vee T\left(x_{n}\right)$. As $\left.T\right|_{X}: X \rightarrow W$ is a Riesz homomorphism, Proposition 2 yields that

$$
\left.\left.T\right|_{X}\left(x_{1}\right) \vee \cdots \vee T\right|_{X}\left(x_{n}\right)=\inf \left\{\left.T\right|_{X}(x) ; x \in X, x \in\left\{x_{1}, \ldots, x_{n}\right\}^{\mathrm{u}}\right\} .
$$

For every $x \in X$ with $x \in\left\{x_{1}, \ldots, x_{n}\right\}^{\mathrm{u}}$, we have $x \geq x_{1} \vee \cdots \vee x_{n}$ in $V$, therefore $T(x) \geq T\left(x_{1} \vee \cdots \vee x_{n}\right)$. It follows that $T\left(x_{1}\right) \vee \cdots \vee T\left(x_{n}\right) \geq T\left(x_{1} \vee \cdots \vee x_{n}\right)$.

Second, observe that $V$ is the Riesz completion of $X$ and denote by $S: V \rightarrow W$ the van Haandel extension of the Riesz* homomorphism $\left.T\right|_{X}$. We show that $T=S$, which is a Riesz homomorphism. Indeed, let $v \in V$. Then there are $a_{1}, \ldots, a_{m}, b_{1}, \ldots, b_{n} \in$ $X$ with $v=\bigvee_{i=1}^{m} a_{i}-\bigvee_{j=1}^{n} b_{j}$. By (1), we obtain 


$$
\begin{aligned}
T v & =T\left(\bigvee_{i=1}^{m} a_{i}\right)-T\left(\bigvee_{j=1}^{n} b_{j}\right)=\bigvee_{i=1}^{m} T\left(a_{i}\right)-\bigvee_{j=1}^{n} T\left(b_{j}\right) \\
& =\bigvee_{i=1}^{m} S\left(a_{i}\right)-\bigvee_{j=1}^{n} S\left(b_{j}\right)=S v .
\end{aligned}
$$

The condition in Proposition 6 that $W$ need to be a Riesz space can be relaxed to $W$ being a pre-Riesz space.

Corollary 7 Let $V$ be a Riesz space, let $W$ be a pre-Riesz space, and let $X$ be an order dense subspace of $V$ that generates $V$ as a Riesz space. Let $T: V \rightarrow W$ be a positive linear operator. If $\left.T\right|_{X}: X \rightarrow W$ is a Riesz homomorphism, then $T$ is a Riesz. homomorphism.

Proof Let $\left(W^{\rho}, i\right)$ be the Riesz completion of $W$. Since $i$ is a complete Riesz homomorphism by [9, Proposition 2.3.27], it follows from [9, Proposition 2.3.24(ii)] that $\left.i \circ T\right|_{X}: X \rightarrow W^{\rho}$ is a Riesz homomorphism. Therefore, Proposition 6 yields that $i \circ T: V \rightarrow W^{\rho}$ is a Riesz homomorphism. With the aid of Proposition 3, it follows that $i \circ T: V \rightarrow i[W]$ is a Riesz homomorphism. As $i$ is an order isomorphism, $T$ is a Riesz homomorphism.

Corollary 8 Consider the setting of Theorem 5. If $T: X_{1} \rightarrow X_{2}$ is a Riesz homomorphism and $S, \tilde{S}: Y_{1} \rightarrow Y_{2}$ are positive linear operators such that $S \circ i_{1}=i_{2} \circ T$ and $\tilde{S} \circ i_{1}=i_{2} \circ T$, then $S=\tilde{S}$. Moreover, $S$ equals the van Haandel extension of $T$.

This corollary has a useful consequence for the van Haandel extensions of sums of operators.

Proposition 9 Let $X_{1}$ and $X_{2}$ be pre-Riesz spaces with Riesz completions $\left(Y_{1}, i_{1}\right)$ and $\left(Y_{2}, i_{2}\right)$, respectively. Let $S, T: X_{1} \rightarrow X_{2}$ be Riesz $*$ homomorphisms with van Haandel extensions $\bar{S}, \bar{T}: Y_{1} \rightarrow Y_{2}$, respectively. If $S+T$ is a Riesz homomorphism, then the van Haandel extension of $S+T$ equals $\bar{S}+\bar{T}$.

Proof Since $\bar{S}+\bar{T}$ is a positive operator extending $S+T$, it follows from Corollary 8 that $\bar{S}+\bar{T}$ equals the van Haandel extension of $S+T$.

For complete Riesz homomorphisms, observe the following result, which is a slightly more general version of [12, Lemma 4.3].

Proposition 10 Let $Y_{1}, Y_{2}$ be partially ordered vector spaces and $X_{1}, X_{2}$ order dense subspaces of $Y_{1}, Y_{2}$, respectively. Let $T: Y_{1} \rightarrow Y_{2}$ be a positive linear operator with $T\left[X_{1}\right] \subseteq X_{2}$. Then $T$ is a complete Riesz homomorphism if and only if $\left.T\right|_{X_{1}}: X_{1} \rightarrow$ $\mathrm{X}_{2}$ is a complete Riesz homomorphism.

Proof First, observe that for $A \subseteq X_{1}$ and $s \in X_{1}$, we have

$$
\inf A=s \text { in } X_{1} \text { if and only if } \inf A=s \text { in } Y_{1} \text {. }
$$


Indeed, assume that $\inf A=s$ in $X_{1}$. If $y \in Y_{1}$ is a lower bound of $A$ in $Y_{1}$, then, as $X_{1}$ is order dense in $Y_{1}$, we have $y=\sup \left\{x \in X_{1} ; x \leq y\right\}$. Every $x$ in the latter set is a lower bound of $A$, hence $x \leq s$, and, therefore, $y \leq s$. The converse implication is straightforward.

Now let $T$ be a complete Riesz homomorphism. Let $A \subseteq X_{1}$ be such that inf $A=0$ in $X_{1}$, then, by (2), inf $A=0$ in $Y_{1}$. As $T$ is a complete Riesz homomorphism, we get inf $T[A]=0$ in $Y_{2}$. Since $T[A] \subseteq X_{2}$, we conclude inf $T[A]=0$ in $X_{2}$, hence $\left.T\right|_{X_{1}}: X_{1} \rightarrow X_{2}$ is a complete Riesz homomorphism.

Conversely, let $\left.T\right|_{X_{1}}: X_{1} \rightarrow X_{2}$ be a complete Riesz homomorphism. Let $A \subseteq Y_{1}$ be such that inf $A=0$ in $Y_{1}$. Define

$$
A_{0}:=\left\{x \in X_{1} ; \exists a \in A: x \geq a\right\} .
$$

Clearly, 0 is a lower bound of $A_{0}$. Let $v \in X_{1}$ be a lower bound of $A_{0}$. For every $a \in A$, as $X_{1}$ is order dense in $Y_{1}$, we have $a=\inf \left\{x \in X_{1} ; x \geq a\right\} \geq v$. Thus, $0=\inf A \geq v$. This implies inf $A_{0}=0$ in $X_{1}$. By assumption, we get inf $T\left[A_{0}\right]=0$ in $X_{2}$, and also in $Y_{2}$ by (2). As $T$ is positive, 0 is a lower bound of $T$ [A] in $Y_{2}$. Let $y \in Y_{2}$ be a lower bound of $T[A]$. Then $y \leq b$ for every $b \in T\left[A_{0}\right]$. Indeed, let $b \in T\left[A_{0}\right]$. Then there is $x \in A_{0}$ such that $b=T(x)$. Further, there is $a \in A$ such that $x \geq a$. Thus, $y \leq T(a) \leq T(x)=b$. We conclude that $y \leq \inf T\left[A_{0}\right]=0$. Therefore, $\inf T[A]=0$ in $Y_{2}$, which means that $T$ is a complete Riesz homomorphism.

Corollary 11 Consider the setting of Theorem 5. If $T: X_{1} \rightarrow X_{2}$ is a complete Riesz homomorphism, then its van Haandel extension is a complete Riesz homomorphism, as well.

\section{Riesz* homomorphisms and complete Riesz homomorphisms on spaces of continuous functions}

For Riesz* homomorphisms on order dense subspaces of spaces of continuous functions, a representation as weighted composition operators (similar to the vector lattice setting) is given by van Imhoff in [12, Theorem 3.2], see also [9, Theorem 5.1.14], which we state in Theorem 12 below. We say that a subspace $X$ of $\mathrm{C}(P)$ strongly separates the points of $P$ if, for every $p_{1}, p_{2} \in P$ with $p_{1} \neq p_{2}$, there is $x \in X$ such that $x\left(p_{1}\right)=0$ and $x\left(p_{2}\right)=1$.

Theorem 12 Let $P$ and $Q$ be nonempty compact Hausdorff spaces and let $X$ and $Y$ be order dense subspaces of $\mathrm{C}(P)$ and $\mathrm{C}(Q)$, respectively. Let $T: X \rightarrow Y$ be linear.

(i) If $T$ is a Riesz ${ }^{*}$ homomorphism, then there exist $w: Q \rightarrow \mathbb{R}_{+}$and $\alpha: Q \rightarrow P$ such that, for every $x \in X$ and $q \in Q$, we have

$$
(T x)(q)=w(q) x(\alpha(q))
$$

If, in addition, $X$ strongly separates the points of $P$, then $w$ can be taken such that $w$ is continuous on $Q$, the map $\alpha$ is uniquely determined on $\{q \in Q ; w(q)>0\}$, and, on this set, $\alpha$ is continuous. 
(ii) If there exist $w \in \mathrm{C}(Q), w \geq 0$, and $\alpha: Q \rightarrow P$ continuous on $\{q \in Q ; w(q)>$ $0\}$ such that (3) holds for every $x \in X$ and $q \in Q$, then $T$ is a Riesz* homomorphism.

Since we will need the statement later on, we extract a part of the proof of Theorem 12 as a proposition, see also [12, Proof of Theorem 3.2].

Proposition 13 Let $P$ and $Q$ be nonempty compact Hausdorff spaces and let $X$ be an order dense subspace of $\mathrm{C}(P)$ that strongly separates the points of $P$. Let $w: Q \rightarrow \mathbb{R}_{+}$ and $\alpha: Q \rightarrow P$ be such that $q \mapsto w(q) x(\alpha(q)) \in \mathrm{C}(Q)$ for every $x \in X$. Then $w$ is continuous on $Q$ and $\alpha$ is continuous on $\{q \in Q ; w(q)>0\}$.

The following result is given in [12, Lemma 4.2].

Lemma 14 Let $P$ be a nonempty compact Hausdorff space and let $X$ be an order dense subspace of $\mathrm{C}(P)$. Let $G \subseteq X$ be a set of positive elements. Then the following statements are equivalent.

(i) $\inf G=0$.

(ii) For every $\varepsilon>0$ and every nonempty open set $U \subseteq P$, there exist $x \in G$ and $p \in U$ such that $x(p) \leq \varepsilon$.

For complete Riesz homomorphisms, the representation as weighted composition operator in Theorem 12 can be specified as follows, see [12, Theorem 4.4]. Hereby, $\alpha: Q \rightarrow P$ is called weak-open if, for every nonempty open set $U \subseteq Q$, the set $\alpha[U]$ is dense somewhere, i.e., there exists a nonempty open set $V \subseteq P$ such that $\alpha[U] \cap V$ is dense in $V$.

Theorem 15 Let $P$ and $Q$ be nonempty compact Hausdorff spaces, let $X$ be an order dense subspace of $\mathrm{C}(P)$ and let $Y$ be a subspace of $\mathrm{C}(Q)$. Let $T: X \rightarrow Y$ be given by (3), where $w \in \mathrm{C}(P), w \geq 0$, and $\alpha: Q \rightarrow P$. Then the following statements are equivalent.

(i) $T$ is a complete Riesz homomorphism.

(ii) $\alpha$ is weak-open on $\{q \in Q ; w(q)>0\}$.

In the setting of the previous theorems, pervasiveness can be characterized as follows, see also [12, Lemma 6.4].

Proposition 16 Let $P$ be a nonempty compact Hausdorff space and $X$ an order dense subspace of $\mathrm{C}(P)$. The space $X$ is pervasive if and only if for every nonempty open set $U \subseteq P$, there exists an $x \in X \backslash\{0\}, x \geq 0$, such that $\operatorname{coz}(x) \subseteq U$, where $\operatorname{coz}(x):=\{u \in P ; x(u) \neq 0\}$.

Proof Let $X$ be pervasive in the vector lattice cover $\mathrm{C}(P)$ and let $U \subseteq P$ be nonempty and open. Let $q \in U$. By Urysohn's lemma, there exists a positive function $y \in \mathrm{C}(P)$ such that $y(q)=1$ and $y=0$ on $P \backslash U$. Hence $\operatorname{coz}(y) \subseteq U$. Since $y \neq 0$ and $X$ is pervasive, there is $x \in X \backslash\{0\}$ with $0 \leq x \leq y$. Therefore, $\operatorname{coz}(x) \subseteq U$.

Conversely, let $y \in \mathrm{C}(P) \backslash\{0\}, y \geq 0$. Fix $\varepsilon>0$ such that $\varepsilon<\|y\|_{\infty}$. Define $U:=$ $\{p \in P ; y(p)>\varepsilon\}$. Then $U$ is nonempty and open, hence there exists $v \in X \backslash\{0\}$, $v \geq 0$, with $\operatorname{coz}(v) \subseteq U$. For $x:=\frac{\varepsilon}{\|v\|_{\infty}} v \in X \backslash\{0\}$, we get $0 \leq x \leq y$, hence $X$ is pervasive. 
Next we consider order unit spaces and recall a characterization of functionals that are Riesz* homomorphisms. Let $(X, K)$ be an Archimedean partially ordered vector space with order unit $u$, equipped with the order unit norm $\|x\|_{u}:=\inf \{\lambda \in$ $(0, \infty) ;-\lambda u \leq x \leq \lambda u\}$ for $x \in X$, see, e.g., [9, Section 1.5.3]. Every order unit space is pre-Riesz. We construct a vector lattice cover with pointwise partial order. The functional representation of $X$ is given by means of the weakly-* compact convex set

$$
\Sigma:=\left\{\varphi \in X^{\prime} ; \varphi[K] \subseteq[0, \infty), \varphi(u)=1\right\}
$$

and the set $\Lambda$ of the extreme points of $\Sigma$. The weak-* closure $\bar{\Lambda}$ of $\Lambda$ in $\Sigma$ is (with the weak-* topology) a compact Hausdorff space, and the map

$$
\Phi: X \rightarrow \mathrm{C}(\bar{\Lambda}), \quad x \mapsto(\varphi \mapsto \varphi(x))
$$

is a bipositive linear map, and hence injective (for details, see, e.g., [9, Section 2.5]). In [8], it is shown that $(\mathrm{C}(\bar{\Lambda}), \Phi)$ is a vector lattice cover of $X$, see also [9, Theorem 2.5.9]. We recall the statement in [9, Proposition 2.5.5].

Proposition 17 Let $X$ be an order unit space and let $\varphi \in \Sigma$.

(a) One has $\varphi \in \Lambda$ if and only if $\varphi$ is a Riesz homomorphism.

(b) One has $\varphi \in \bar{\Lambda}$ if and only if $\varphi$ is a Riesz* homomorphism.

Example 18 (Lorentz cone) Let $H$ be a Hilbert space and let $X:=\mathbb{R} \times H$ be ordered by the Lorentz cone $L_{H}:=\left\{(r, z) \in \mathbb{R} \times H ; r^{2}-\langle z, z\rangle \geq 0\right.$ and $\left.r \geq 0\right\}$. We deal with the functional representation with respect to the order unit $u:=(1,0)$, for details, see [9, Lemma 2.6.3 and Theorem 2.6.4]. If $H$ is $n$-dimensional, then we have $\bar{\Lambda}=\Lambda$, and $\Lambda$ can be identified with the $(n-1)$-sphere in $\mathbb{R}^{n}$. In this case, by Proposition 17 , the set $\bar{\Lambda}$ in (5) consists of Riesz homomorphisms.

Example 19 (Polyhedral cone) Let $X:=\mathbb{R}^{n}$ and let $K$ be a generating polyhedral cone in $\mathbb{R}^{n}$. Then there is an order unit $u$ in $K$, and there are $k \geq n$ linear functionals $f^{(1)}, \ldots, f^{(k)}$ such that

$$
K=\left\{x \in \mathbb{R}^{n} ; \forall i \in\{1, \ldots, k\}: f^{(i)}(x) \geq 0\right\}
$$

where each $f^{(i)}$ defines a facet ${ }^{1}$ of $K$ and $f^{(i)}(u)=1$; for details, see also [9, Section 2.6.1]. In view of (5), we get

$$
\bar{\Lambda}=\Lambda=\left\{f^{(1)}, \ldots, f^{(k)}\right\}
$$

and $\Phi:\left(\mathbb{R}^{n}, K\right) \rightarrow\left(\mathbb{R}^{k}, \mathbb{R}_{+}^{k}\right)$

$$
\Phi(x):=\left(f_{1}(x), \ldots, f_{k}(x)\right)^{\mathrm{T}} \quad \text { for } x \in \mathbb{R}^{n} .
$$

$\left.\left.\overline{1 \text { This means } \operatorname{dim}\left(\left\{x \in \mathbb{R}^{n} ; f^{(i)}\right.\right.}(x)=0\right\}\right)=n-1$. 
In [8], it is shown that $\left(\mathbb{R}^{k}, \Phi\right)$ is the Riesz completion of $\left(\mathbb{R}^{n}, K\right)$, see also [9, Theorem 2.6.2].

Lemma 20 Let $X$ be an order unit space, where $u$ is an order unit, and $(\mathrm{C}(\bar{\Lambda}), \Phi)$ its functional representation corresponding to (5). Then $\Phi[X]$ strongly separates the points of $\bar{\Lambda}$.

Proof Let $\varphi_{1}, \varphi_{2} \in \bar{\Lambda}$ with $\varphi_{1} \neq \varphi_{2}$. Then there is $z \in X$ with $\varphi_{1}(z) \neq \varphi_{2}(z)$. We have that $\varphi_{1}(u)=\varphi_{2}(u)=1$. We define

$$
x:=\frac{z-\varphi_{1}(z) u}{\varphi_{2}(z)-\varphi_{1}(z)}
$$

and observe that $(\Phi(x))\left(\varphi_{1}\right)=\varphi_{1}(x)=0$ and $(\Phi(x))\left(\varphi_{2}\right)=\varphi_{2}(x)=1$. Thus, $\Phi[X]$ strongly separates the points of $\bar{\Lambda}$.

Remark 21 Let $X$ be an order unit space and let $v$ be an order unit in $X$. If $w \in X$ is such that $w \perp v$, then $w=0$. Indeed, using the functional representation, we have $(\Phi(v))(\varphi)>0$ for every $\varphi \in \bar{\Lambda}$. As $\Phi(v) \perp \Phi(w)$, we get $\Phi(w)=0$ and, hence, $w=0$.

\section{Characterization of functionals that are complete Riesz homomorphisms}

In the spirit of Proposition 17, we characterize those functionals on order unit spaces that are complete Riesz homomorphisms. First, we study point evaluations that are complete Riesz homomorphisms.

Lemma 22 Let $P$ be a nonempty compact Hausdorff space, let $p \in P$, and let $\varphi: \mathrm{C}(P) \rightarrow \mathbb{R}$ be given by $\varphi(x)=x(p)$ for $x \in \mathrm{C}(P)$. Then $\varphi$ is a complete Riesz homomorphism if and only if $\{p\}$ is open in $P$.

Proof We view $\varphi$ as a map from $\mathrm{C}(P)$ to $\mathrm{C}(Q)$, where the set $Q$ consists of one point $q$, and, for every $x \in \mathrm{C}(Q)$, the real number $\varphi(x)$ is interpreted as the continuous function $q \mapsto \varphi(x)$ on $Q$. Define $\alpha: Q \rightarrow P$ by $\alpha(q):=p$. Then, for every $x \in \mathrm{C}(P)$, we have

$$
(\varphi(x))(q)=x(p)=x(\alpha(q))
$$

We show that $\alpha$ is weak-open if and only if $\{p\}$ is open in $P$. For a proof, assume first that $\alpha$ is weak-open. Then $\alpha[Q]=\{\alpha(q)\}=\{p\}$ is dense somewhere, that is, there is a nonempty open set $V \subseteq P$ such that $\{p\} \cap V$ is dense in $V$. We obtain that $V=\{p\}$. Indeed, if $p \notin V$, then $\{p\} \cap V=\varnothing$ is not dense in $V$ as $V$ is nonempty. If there is $p_{0} \in V \backslash\{p\}$, then there is a net in $\{p\}$ converging to $p_{0}$, which contradicts the fact that $P$ is Hausdorff. We conclude that $\{p\}$ is open in $P$.

Conversely, assume that $\{p\}$ is open in $P$. Let $U \subseteq Q$ be nonempty and open. Then $U=\{q\}$, so $\alpha[U]=\{\alpha(q)\}=\{p\}$. We thus have that $\{p\}$ is open and nonempty and 
$\alpha[U] \cap\{p\}=\{p\}$ is dense in $\{p\}$. Hence, $\alpha$ is weak-open. Now, the requested claim follows from Theorem 15 .

Example 23 It is well-known that for every $t \in[-1,1]$ the point evaluation $\varphi_{t}: \mathrm{C}[-1,1] \rightarrow \mathbb{R}, x \mapsto \varphi_{t}(x):=x(t)$, is a Riesz homomorphism, but not order continuous. Hence, it is not a complete Riesz homomorphism, as is also indicated by Lemma 22. Observe that point evaluations on order dense subspaces of $C[-1,1]$ are not Riesz homomorphisms, in general. For instance, the Namioka space $X$ in Example 42 below is order dense in $C[-1,1]$, but the restriction of $\varphi_{0}$ to $X$ is not a Riesz homomorphism, see Proposition 17.

The next statement provides the characterization of functionals that are complete Riesz homomorphisms.

Proposition 24 Let $\left(X, K,\|\cdot\|_{u}\right)$ be an order unit space and let $(\mathrm{C}(\bar{\Lambda})$, $\Phi)$ be its functional representation according to (5). Then $\varphi \in \bar{\Lambda}$ is a complete Riesz homomorphism if and only if $\{\varphi\}$ is open in $\bar{\Lambda}$.

Proof By Lemma 22, the set $\{\varphi\}$ is open in $\bar{\Lambda}$ if and only if the point evaluation $\hat{\varphi}: \mathrm{C}(\bar{\Lambda}) \rightarrow \mathbb{R}, x \mapsto x(\varphi)$, is a complete Riesz homomorphism. This is equivalent to $\hat{\varphi} \circ \Phi=\varphi$ being a complete Riesz homomorphism, due to Proposition 10 .

Example 25 (Polyhedral cone) In Example 19, the set $\bar{\Lambda}=\Lambda$ consists of complete Riesz homomorphisms. Indeed, for every $\varphi \in \Lambda$, we have that $\{\varphi\}$ is open in $\Lambda$, thus $\varphi$ is a complete Riesz homomorphism by Proposition 24.

In Riesz spaces, a linear map is a complete Riesz homomorphism if and only if it is an order continuous Riesz homomorphism, see, e.g., [9, Proposition 1.4.5]. In pre-Riesz spaces, every complete Riesz homomorphism is an order continuous Riesz homomorphism, see [9, Propositions 2.3.16 and 2.3.20]. We give a functional on a pre-Riesz space that is an order continuous Riesz homomorphism, but not a complete Riesz homomorphism.

Example 26 (Lorentz cone) We continue Example 18 with $H$ being an $n$-dimensional Hilbert space, $X:=\mathbb{R} \times H$ and $L_{H}$ the Lorentz cone in $X$. Let $\varphi \in \Lambda=\bar{\Lambda}$. We already observed that $\varphi$ is a Riesz homomorphism. As $\Lambda$ can be identified with the $(n-1)$-sphere in $\mathbb{R}^{n}$, the set $\{\varphi\}$ is not open. Proposition 24 yields that $\varphi$ is not a complete Riesz homomorphism. By Proposition 4, the map $\varphi$ is order continuous.

\section{Riesz homomorphisms and complete Riesz homomorphisms on order unit spaces}

By means of the functional representation, we characterize in Theorem 29 the order unit spaces on which every Riesz* homomorphism is a Riesz homomorphism, or a complete Riesz homomorphism, respectively. First, we consider operators that are represented as weighted composition operators as in Theorem 12. As preparatoy work for Theorem 29, we prove the following technical proposition, whose main content is part (i), since part (ii) follows from Theorem 15. Nevertheless, the consequence in Theorem 29(ii) below has not been observed, so far. 
Proposition 27 Let $\left(X_{1}, K_{1},\|\cdot\|_{u}\right), \quad\left(X_{2}, K_{2},\|\cdot\|_{v}\right)$ be order unit spaces and $\left(\mathrm{C}\left(\overline{\Lambda_{1}}\right), \Phi_{1}\right),\left(\mathrm{C}\left(\overline{\Lambda_{2}}\right), \Phi_{2}\right)$ their functional representations, respectively. Let $w \in$ $\mathrm{C}\left(\overline{\Lambda_{2}}\right)$ be positive and $\alpha: \overline{\Lambda_{2}} \rightarrow \overline{\Lambda_{1}}$. Let the linear operator $T_{0}: \Phi_{1}\left[X_{1}\right] \rightarrow \mathrm{C}\left(\overline{\Lambda_{2}}\right)$ be such that $T_{0}\left[\Phi_{1}\left[X_{1}\right]\right] \subseteq \Phi_{2}\left[X_{2}\right]$ and given by

$$
\left(T_{0} v\right)(q)=w(q) v(\alpha(q)) \quad \text { for } v \in \Phi_{1}\left[X_{1}\right], q \in \overline{\Lambda_{2}} \text {. }
$$

If we define the linear operator $T: X_{1} \rightarrow X_{2}$ by $T:=\Phi_{2}^{-1} \circ T_{0} \circ \Phi_{1}$, then the following statements hold.

(i) If, for every nonempty open set $U \subseteq\left\{q \in \overline{\Lambda_{2}} ; w(q)>0\right\}$, there exists $q \in U$ such that $\alpha(q) \in \Lambda_{1}$, then $T$ is a Riesz homomorphism.

(ii) If, for every nonempty open set $U \subseteq\left\{q \in \overline{\Lambda_{2}} ; w(q)>0\right\}$, there exists $q \in U$ such that $\{\alpha(q)\}$ is open in $\overline{\Lambda_{1}}$, then $T$ is a complete Riesz homomorphism.

Proof (i) We show that $T_{0}$ is a Riesz homomorphism. We intend to apply Proposition 3 with $Y:=\mathrm{C}\left(\overline{\Lambda_{2}}\right)$. Let $x, y \in \Phi_{1}\left[X_{1}\right]$ and denote

$$
\begin{aligned}
G: & =\left\{T_{0} v-\left(T_{0} x \vee T_{0} y\right) ; v \in \Phi_{1}\left[X_{1}\right], v \in\{x, y\}^{\mathrm{u}}\right\} \\
& =\left\{q \mapsto w(q)(v(\alpha(q))-x(\alpha(q)) \vee y(\alpha(q))) ; v \in \Phi_{1}\left[X_{1}\right], v \in\{x, y\}^{\mathrm{u}}\right\} \\
& \subseteq Y .
\end{aligned}
$$

As $T_{0}$ is positive, the set $G$ consists of positive elements. We show that inf $G=0$ in $\mathrm{C}\left(\overline{\Lambda_{2}}\right)$ using Lemma 14 with $X:=\mathrm{C}\left(\overline{\Lambda_{2}}\right)$. Let $\varepsilon>0$ and $U \subseteq \overline{\Lambda_{2}}$ be a nonempty open set. We investigate two cases. First, assume that there is $q_{0} \in U$ such that $w\left(q_{0}\right)=0$. As $\Phi_{1}\left[X_{1}\right]$ is directed, there is $v \in \Phi_{1}\left[X_{1}\right]$ such that $v \geq x$ and $v \geq y$. Now

$$
w\left(q_{0}\right)\left(v\left(\alpha\left(q_{0}\right)\right)-x\left(\alpha\left(q_{0}\right)\right) \vee y\left(\alpha\left(q_{0}\right)\right)\right)=0<\varepsilon .
$$

Second, we consider the case $U \subseteq\left\{q \in \overline{\Lambda_{2}} ; w(q)>0\right\}$. By assumption, there exists $q_{0} \in U$ such that $\alpha\left(q_{0}\right) \in \Lambda_{1}$. By Proposition 17, the functional $\alpha\left(q_{0}\right)$ is a Riesz homomorphism on $X_{1}$. Since $\Phi_{1}^{-1}: \Phi_{1}\left[X_{1}\right] \rightarrow X_{1}$ is an order isomorphism, we have that $\varphi:=\alpha\left(q_{0}\right) \circ \Phi_{1}^{-1}: \Phi_{1}\left[X_{1}\right] \rightarrow \mathbb{R}$ is a Riesz homomorphism, and therefore,

$$
\inf \left\{\varphi(v) ; v \in \Phi_{1}\left[X_{1}\right], v \in\{x, y\}^{\mathrm{u}}\right\}=\varphi(x) \vee \varphi(y)
$$

Let $M>0$ be an upper bound of $w$. Then there exists $v \in \Phi_{1}\left[X_{1}\right]$ with $v \in\{x, y\}^{\mathrm{u}}$ such that

$$
\varphi(v) \leq \varphi(x) \vee \varphi(y)+\frac{\varepsilon}{M} .
$$

Recall that for every $a \in X_{1}$ and $\lambda \in \overline{\Lambda_{1}}$, we have $\left(\Phi_{1}(a)\right)(\lambda)=\lambda(a)$. Hence, for $z \in \Phi_{1}\left[X_{1}\right]$, the choice $a:=\Phi_{1}^{-1}(z)$ and $\lambda:=\alpha\left(q_{0}\right)$ yields

$$
\varphi(z)=\alpha\left(q_{0}\right)\left(\Phi_{1}^{-1}(z)\right)=\Phi_{1}\left(\Phi_{1}^{-1}(z)\right)\left(\alpha\left(q_{0}\right)\right)=z\left(\alpha\left(q_{0}\right)\right) .
$$


Now, (9) reads as

$$
v\left(\alpha\left(q_{0}\right)\right) \leq x\left(\alpha\left(q_{0}\right)\right) \vee y\left(\alpha\left(q_{0}\right)\right)+\frac{\varepsilon}{M},
$$

which implies

$$
\begin{aligned}
w\left(q_{0}\right)\left(v\left(\alpha\left(q_{0}\right)\right)-x\left(\alpha\left(q_{0}\right)\right) \vee y\left(\alpha\left(q_{0}\right)\right)\right) & \leq M\left(v\left(\alpha\left(q_{0}\right)\right)-x\left(\alpha\left(q_{0}\right)\right) \vee y\left(\alpha\left(q_{0}\right)\right)\right) \\
& \leq \varepsilon .
\end{aligned}
$$

We conclude that in both cases condition (ii) in Lemma 14 is satisfied, hence inf $G=0$. By Proposition 3, it follows that $T_{0}$ is a Riesz homomorphism. As $T=\Phi_{2}^{-1} \circ T_{0} \circ \Phi_{1}$ and $\Phi_{1}, \Phi_{2}^{-1}$ are order isomorphisms, $T$ is a Riesz homomorphism.

(ii) Similar to the proof of (i), we show that $T_{0}$ is a complete Riesz homomorphism. Let $A \subseteq \Phi_{1}\left[X_{1}\right]$ be a set of positive elements such that inf $A=0$. We intend to show that inf $T_{0}[A]=0$ using Lemma 14 for $G:=T_{0}[A]$. Clearly, the set $G$ consists of positive elements. Let $\varepsilon>0$ and $U \subseteq \overline{\Lambda_{2}}$ be a nonempty open set. First, if there is $q_{0} \in U$ such that $w\left(q_{0}\right)=0$, then, for every $v \in A$, we have

$$
T_{0}(v)\left(q_{0}\right)=w\left(q_{0}\right) v\left(\alpha\left(q_{0}\right)\right)=0<\varepsilon .
$$

Second, let $U \subseteq\left\{q \in \overline{\Lambda_{2}} ; w(q)>0\right\}$. By assumption, there exists $q_{0} \in U$ such that $\left\{\alpha\left(q_{0}\right)\right\}$ is open is $\overline{\Lambda_{1}}$. By Proposition 24, $\alpha\left(q_{0}\right)$ is a complete Riesz homomorphism on $X_{1}$, hence $\varphi:=\alpha\left(q_{0}\right) \circ \Phi_{1}^{-1}: \Phi_{1}\left[X_{1}\right] \rightarrow \mathbb{R}$ is a complete Riesz homomorphism, and therefore, $\inf \varphi[A]=0$. Let $M>0$ be an upper bound of $w$. Then there exists $v \in A$ such that

$$
v\left(\alpha\left(q_{0}\right)\right)=\varphi(v) \leq \frac{\varepsilon}{M}
$$

which implies

$$
T_{0}(v)\left(q_{0}\right)=w\left(q_{0}\right) v\left(\alpha\left(q_{0}\right)\right) \leq M v\left(\alpha\left(q_{0}\right)\right) \leq \varepsilon .
$$

We conclude that condition (ii) in Lemma 14 is satisfied, hence inf $T_{0}[A]=0$. It follows that $T_{0}$ and, therefore, $T$ is a complete Riesz homomorphism.

The sufficient condition in Proposition 27(ii) is not a necessary one. Indeed, consider $X_{1}=X_{2}:=\mathrm{C}[0,1]$ and the identity map $T: x \mapsto x$. Then $T$ is a complete Riesz homomorphism, but, as singletons in $[0,1]$ are not open, the condition in Proposition 27(ii) is not satisfied.

The following digression to rank-one operators will be helpful later on.

Proposition 28 Let $X$ and $Y$ be partially ordered vector spaces, let $v \in Y \backslash\{0\}, v \geq 0$, and let $f: X \rightarrow \mathbb{R}$ be linear. Define the linear map $T: X \rightarrow Y$ by $T x=f(x) v$ for $x \in X$.

(i) $T$ is positive if and only if $f$ is positive.

(ii) $T$ is a Riesz* homomorphism if and only if $f$ is a Riesz* homomorphism. 
(iii) If $T$ is a Riesz homomorphism, then $f$ is a Riesz homomorphism. The converse implication is satisfied if $Y$ is Archimedean.

(iv) If T is a complete Riesz homomorphism, then $f$ is a complete Riesz homomorphism. The converse implication is satisfied if $Y$ is Archimedean.

\section{Proof}

(i) is clear.

(ii) Let $T$ be a Riesz* homomorphism. Let $F \subseteq X$ be a nonempty finite set. Then $T\left[F^{\mathrm{ul}}\right] \subseteq T[F]^{\mathrm{ul}}$. Let $\alpha \in f\left[F^{\mathrm{ul}}\right]$ and let $z \in F^{\mathrm{ul}}$ be such that $f(z)=\alpha$. Define $\mu:=\max \{f(a) ; a \in F\}$. For every $a \in F$, we have $T a=f(a) v \leq \mu v$, hence $\mu v \in T[F]^{\mathrm{u}}$. As $T z \in T\left[F^{\mathrm{ul}}\right] \subseteq T[F]^{\mathrm{ul}}$, we obtain $f(z) v=T z \leq \mu v$. Hence, $f(z) \leq \mu$, and, therefore, $f(z) \in f[F]^{\mathrm{ul}}$. Thus, $f\left[F^{\mathrm{ul}}\right] \subseteq f[F]^{\mathrm{ul}}$, which yields that $f$ is a Riesz* homomorphism.

Conversely, let $f$ be a Riesz* homomorphism. Let $F \subseteq X$ be a nonempty finite set. Then $f\left[F^{\mathrm{ul}}\right] \subseteq f[F]^{\mathrm{ul}}$. Let $y \in T\left[F^{\mathrm{ul}}\right]$ and let $z \in F^{\mathrm{ul}}$ be such that $T z=y$. Let $u \in T[F]^{\mathrm{u}}$. Since $f(z) \in f\left[F^{\mathrm{ul}}\right] \subseteq f[F]^{\mathrm{ul}}$ and $\mu:=\max \{f(a) ; a \in F\} \in$ $f[F]^{\mathrm{u}}$, we have $f(z) \leq \mu$, hence $T z=f(z) v \leq \mu v$. As, for every $a \in F$, $f(a) v=T a \leq u$, we obtain $y=T z \leq \mu v \leq u$. Thus, $y \in T[F]^{\mathrm{ul}}$, which implies that $T$ is a Riesz* homomorphism.

(iii) We use Proposition 2(ii). Suppose that $f$ is positive and not a Riesz homomorphism, then there exist $a, b \in X$ and $\varepsilon>0$ such that

$$
\inf \left\{f(z) ; z \in\{a, b\}^{\mathrm{u}}\right\}>f(a) \vee f(b)+\varepsilon=: \alpha .
$$

For every $z \in\{a, b\}^{\mathrm{u}}$, we have $T z=f(z) v \geq \alpha v$, hence $\alpha v \in T\left[\{a, b\}^{\mathrm{u}}\right]^{1}$. As $\{T a, T b\}^{\mathrm{ul}}=\{f(a) v, f(b) v\}^{\mathrm{ul}}=\{(f(a) \vee f(b)) v\}^{1}$, it follows that $\alpha v \notin\{T a, T b\}^{\mathrm{ul}}$. Hence $T\left[\{a, b\}^{\mathrm{u}}\right]^{\mathrm{l}} \nsubseteq\{T a, T b\}^{\mathrm{ul}}$, which means that $T$ is not a Riesz homomorphism.

Conversely, assume that $Y$ is Archimedean and that $f$ is a Riesz homomorphism. Let $a, b \in X$. By (i), $T$ is positive, hence $\{T a, T b\}^{\mathrm{ul}} \subseteq T\left[\{a, b\}^{\mathrm{u}}\right]^{1}$. By Proposition 2(ii), we have

$$
\mu:=\max \{f(a), f(b)\}=\inf \left\{f(z) ; z \in\{a, b\}^{\mathrm{u}}\right\} .
$$

Let $y \in T\left[\{a, b\}^{\mathrm{u}}\right]^{\mathrm{l}}$ and $u \in\{T a, T b\}^{\mathrm{u}}$. For every $z \in\{a, b\}^{\mathrm{u}}$, we have $y \leq$ $T z=f(z) v$, hence $y \leq \mu v$, as $Y$ is Archimedean, see [16, Theorem 9.1]. As $u \geq f(a) v$ and $u \geq f(b) v$, we get $u \geq \mu v$. We conclude $u \geq y$, which means $T\left[\{a, b\}^{\mathrm{u}}\right]^{1} \subseteq\{T a, T b\}^{\mathrm{ul}}$. Therefore, $T$ is a Riesz homomorphism.

(iv) Let $T$ be a complete Riesz homomorphism. Let $A \subseteq X$ be a nonempty set with $\inf A=0$. Then $\inf \{f(a) v ; a \in A\}=\inf T[A]=0$. This implies inf $f[A]=0$, hence $f$ is a complete Riesz homomorphism.

Conversely, let $f$ be a complete Riesz homomorphism. Then, for a nonempty set $A \subseteq X$ with $\inf A=0$, we get inf $f[A]=0$, therefore inf $T[A]=$ $\inf \{f(a) v ; a \in A\}=0$, as $Y$ is Archimedean. Thus, $T$ is a complete Riesz homomorphism. 
A topological vector space $M$ is called discrete if, for every $x \in M$, the set $\{x\}$ is open. The subsequent statements are convenient if one studies examples.

Theorem $29 \operatorname{Let}\left(X_{1}, K_{1},\|\cdot\|_{u}\right),\left(X_{2}, K_{2},\|\cdot\|_{v}\right)$ be order unit spaces and $\left(\mathrm{C}\left(\overline{\Lambda_{1}}\right), \Phi_{1}\right)$, $\left(\mathrm{C}\left(\overline{\Lambda_{2}}\right), \Phi_{2}\right)$ their functional respresentations, respectively.

(i) One has $\overline{\Lambda_{1}}=\Lambda_{1}$ if and only if every Riesz $*$ homomorphism $T: X_{1} \rightarrow X_{2}$ is a Riesz homomorphism.

(ii) $\overline{\Lambda_{1}}$ is discrete if and only if every Riesz ${ }^{*}$ homomorphism $T: X_{1} \rightarrow X_{2}$ is a complete Riesz homomorphism.

Proof (i) Let $T: X_{1} \rightarrow X_{2}$ be a Riesz* homomorphism. By Theorem 12(i) and Lemma 20, there exist a positive function $w \in \mathrm{C}\left(\overline{\Lambda_{2}}\right)$ and $\alpha: \overline{\Lambda_{2}} \rightarrow \overline{\Lambda_{1}}$ such that the operator $T_{0}:=\Phi_{2} \circ T \circ \Phi_{1}^{-1}$ is given by (8). If $\overline{\Lambda_{1}}=\Lambda_{1}$, then the condition in Proposition 27(i) is trivially met, and we conclude that $T$ is a Riesz homomorphism. Conversely, let $\varphi \in \overline{\Lambda_{1}}$ and consider

$$
T: X_{1} \rightarrow X_{2}, \quad x \mapsto \varphi(x) v
$$

By the Propositions 17(b) and 28(ii), the operator $T$ is a Riesz* homomorphism. The assumption yields that $T$ is a Riesz homomorphism, which implies that $\varphi$ is a Riesz homomorphism, by Proposition 28(iii). Hence, by Proposition 17(a), we get $\varphi \in \Lambda_{1}$.

(ii) Similar to (i), if $\overline{\Lambda_{1}}$ is discrete, then the condition in Proposition 27(ii) is satisfied, hence $T$ is a complete Riesz homomorphism. Conversely, let $\varphi \in \overline{\Lambda_{1}}$ and consider $T$ given by (10). As in (i), the operator $T$ is a Riesz* homomorphism. By assumption, $T$ is a complete Riesz homomorphism. Applying Proposition 28(iv) yields that $\varphi$ is a complete Riesz homomorphism. Hence, by Proposition 24, the set $\{\varphi\}$ is open in $\overline{\Lambda_{1}}$.

Example 30 (Lorentz cone) We continue Example 18, i.e., $H$ is a finite-dimensional Hilbert space, and $X_{1}:=\mathbb{R} \times H$ is ordered by the Lorentz cone $L_{H}$. As $\overline{\Lambda_{1}}=\Lambda_{1}$, Theorem 29(i) yields that every Riesz* homomorphism from $X_{1}$ into an arbitrary order unit space $X_{2}$ is a Riesz homomorphism.

In view of Example 25, we obtain the following consequence of Theorem 29(ii).

Corollary 31 Let $X_{1}=\mathbb{R}^{n}$ be equipped with a generating polyhedral cone and let $X_{2}$ be an order unit space. Then every Riesz* homomorphism $T: X_{1} \rightarrow X_{2}$ is a complete Riesz homomorphism.

In Proposition 28(iii) and (iv), the converse implication is not true, in general, if $Y$ is not Archimedean, as the subsequent examples show.

Example 32 Let $X:=\mathbb{R} \times H$ as in Example 18, where $H:=\mathbb{R}^{2}$. Let $X$ be ordered by the Lorentz cone $L_{H}$, which is a circular cone here. For $x \in X$, instead of $\left(x_{3},\left(x_{1}, x_{2}\right)^{\mathrm{T}}\right)$, we write $\left(x_{1}, x_{2}, x_{3}\right)^{\mathrm{T}}$, as usual. Let $f: X \rightarrow \mathbb{R}, x \mapsto\left\langle x,(1,0,1)^{\mathrm{T}}\right\rangle$. 
By Example 18, the functional $f$ is a Riesz homomorphism. Let $Y:=\mathbb{R}^{2}$ be ordered by the lexicographical cone

$$
C:=\left\{\left(x_{1}, x_{2}\right)^{\mathrm{T}} \in \mathbb{R}^{2} ; x_{1}>0 \text { or } x_{1}=0 \text { and } x_{2} \geq 0\right\} .
$$

Fix $v:=(1,0)^{\mathrm{T}}$ and define $T: X \rightarrow Y, x \mapsto f(x) v$. Let $a:=(0,1,0)^{\mathrm{T}}$ and $b:=(0,-1,0)^{\mathrm{T}}$. Then $f(a)=f(b)=0$, hence $T a=T b=0$, which implies $\{T a, T b\}^{\mathrm{ul}}=\{0\}^{1}$. Let $x=\left(x_{1}, x_{2}, x_{3}\right)^{\mathrm{T}}$ be an upper bound of $a$ and $b$, then $x_{3}> \pm x_{1}$, hence $f(x)>0$. In particular, for $\alpha \in(0, \infty)$, the element $x:=\left(-\frac{1}{\alpha}, 0, \alpha+\frac{1}{\alpha}\right)$ is an upper bound of $a$ and $b$, where $f(x)=\alpha$. Therefore, $f\left[\{a, b\}^{\mathrm{u}}\right]=(0, \infty)$. We conclude $T\left[\{a, b\}^{\mathrm{u}}\right]=\{\lambda v ; \lambda \in(0, \infty)\}$, and $(0,1)^{\mathrm{T}}$ is a lower bound of this set, but $(0,1)^{\mathrm{T}} \notin\{0\}^{1}$. Consequently, $T\left[\{a, b\}^{\mathrm{u}}\right]^{1} \nsubseteq\{T a, T b\}^{\mathrm{ul}}$, which implies that $T$ is not a Riesz homomorphism.

Example 33 Let $X:=\mathbb{R}^{3}$ be ordered by the four-ray cone

$$
K:=\operatorname{pos}\left\{\left(\begin{array}{l}
1 \\
0 \\
1
\end{array}\right),\left(\begin{array}{l}
0 \\
1 \\
1
\end{array}\right),\left(\begin{array}{c}
-1 \\
0 \\
1
\end{array}\right),\left(\begin{array}{c}
0 \\
-1 \\
1
\end{array}\right)\right\} \text {. }
$$

Considering (6), the cone $K$ is given by the four functionals

$$
f^{(1)}=(-1,-1,1), f^{(2)}=(1,-1,1), f^{(3)}=(1,1,1), f^{(4)}=(-1,1,1),
$$

where each of them is a complete Riesz homomorphism by Example 25. Consider $u:=(0,0,1)^{\mathrm{T}} \in K$ and $A:=\{\lambda u ; \lambda \in(0, \infty)\}$. Then inf $A=0$ in $X$. Let $Y, C$, and $v$ be as in Example 32. The set

$$
T[A]:=\left\{f^{(1)}(a) v ; a \in A\right\}=\{\lambda v ; \lambda \in(0, \infty)\}
$$

has $(0,1)^{\mathrm{T}}$ as a lower bound, hence 0 is not the infimum of $T[A]$ in $Y$. Therefore, $T$ is not a complete Riesz homomorphism.

\section{Characterization of elements with modulus}

If a partially ordered vector space $(X, K)$ is an anti-lattice, an element $x \in X$ has a modulus if and only if $x \in K \cup(-K)$. In [8], it is shown that a partially ordered vector space is an anti-lattice if and only if there are no nontrivial positive disjoint elements in the space, see also [9, Theorem 4.1.10]. In this spirit, we characterize elements with modulus in an arbitrary pre-Riesz space in Theorem 35 below.

Lemma 34 Let $(X, K)$ be a pre-Riesz space and let $(Y, i)$ be a vector lattice cover of $X$. If $x \in X$ is such that $|x|:=\sup \{x,-x\}$ exists in $X$, then

(a) $i(|x|)=|i(x)|$, and 
(b) there exist $a, b \in K, a \perp b$, such that $x=a-b$.

Proof (a) Let $x \in X$ be such that $|x|$ exists in $X$. As $\pm x \leq|x|$, we get $\pm i(x) \leq i(|x|)$ and, hence, $|i(x)| \leq i(|x|)$. By order denseness of $i[X]$ in $Y$, we have

$$
\begin{aligned}
|i(x)| & =\inf \{i(w) ; w \in X, i(w) \geq|i(x)|\} \\
& =\inf \{i(w) ; w \in X, w \geq \pm x\} .
\end{aligned}
$$

If $w \in X$ is such that $w \geq \pm x$, then $w \geq|x|$, so $i(w) \geq i(|x|)$. By (13), it follows that $|i(x)| \geq i(|x|)$. Hence, $|i(x)|=i(|x|)$.

(b) Let $x \in X$ be such that $|x|$ exists in $X$. Define $a:=\frac{1}{2}(x+|x|)$ and $b:=a-x$. Using (a), we get

$$
\begin{aligned}
i(a) & =\frac{1}{2}(i(x)+i(|x|))=\frac{1}{2}(i(x)+|i(x)|) \\
& =\frac{1}{2}\left(i(x)^{+}-i(x)^{-}+i(x)^{+}+i(x)^{-}\right)=i(x)^{+}, \\
i(b) & =i(a)-i(x)=i(x)^{+}-\left(i(x)^{+}-i(x)^{-}\right)=i(x)^{-} .
\end{aligned}
$$

Therefore, $i(a)$ and $i(b)$ are positive disjoint elements in $Y$. We conclude that $a$ and $b$ are positive disjoint elements in $X$, and we have $x=a-b$.

Theorem 35 Let $(X, K)$ be a pre-Riesz space and let $(Y, i)$ be a vector lattice cover of $X$. For $x \in X$, the following statements are equivalent.

(i) $|x|$ exists in $X$.

(ii) $|i(x)| \in i[X]$.

(iii) Either ${ }^{2} x \in K \cup(-K)$, or there exist $a, b \in K \backslash\{0\}, a \perp b$, such that $x \in$ $\operatorname{span}\{a, b\}$.

(iv) Either $x \in K \cup(-K)$, or there exists a subspace $D$ of $X$ with $x \in D$ and $i[D]$ is a Riesz subspace of $Y$.

Proof (i) $\Rightarrow$ (iii): Let $x \in X \backslash(K \cup(-K))$ be such that $|x|$ exists in $X$. By Lemma 34(b), there exist $a, b \in K, a \perp b$, such that $x=a-b$. As $x \notin K \cup(-K)$, it follows that $a \neq 0, b \neq 0$.

(iii) $\Rightarrow$ (iv): Let $x \in X \backslash(K \cup(-K))$ and let $a_{1}, a_{2}$ be nontrivial positive disjoint elements with $x \in \operatorname{span}\left\{a_{1}, a_{2}\right\}$. Define $D:=\operatorname{span}\left\{a_{1}, a_{2}\right\}$. Let $y \in i[D]$, i.e., there are real numbers $\alpha_{1}, \alpha_{2}$ such that $y=\alpha_{1} i\left(a_{1}\right)+\alpha_{2} i\left(a_{2}\right)$. Let $P:=\left\{j \in\{1,2\} ; \alpha_{j} \geq 0\right\}$ and define

$$
y_{1}:=\sum_{p \in P} \alpha_{p} i\left(a_{p}\right) \text { and } y_{2}:=\sum_{p \in\{1,2\} \backslash P}\left|\alpha_{p}\right| i\left(a_{p}\right) .
$$

Then $y_{1} \geq 0, y_{2} \geq 0, y_{1} \perp y_{2}$, and $y=y_{1}-y_{2}$. Hence $y_{1}=y^{+}$and $y_{2}=y^{-}$, and these elements are in $i[D]$. We conclude that $i[D]$ is a Riesz subspace of $Y$.

\footnotetext{
${ }^{2}$ Clearly, (iii) can also be formulated as follows: There exist disjoint elements $a, b \in K$ such that $x \in$ $\operatorname{span}\{a, b\}$. However, this formulation might hide the geometry of the problem.
} 
(iv) $\Rightarrow$ (ii): If $x \in D$ for a subspace $D$ of $X$ such that $i[D]$ is a Riesz subspace of $Y$, we have $|i(x)| \in i[D] \subseteq i[X]$.

(ii) $\Rightarrow$ (i): Let $x \in X$ and assume that there is an element $v \in X$ such that $|i(x)|=$ $i(v)$. We show that $v=|x|$. Since $i(v) \geq \pm i(x)$, it follows that $v \geq \pm x$, due to the bipositivity of $i$. Moreover, $w \geq \pm x$ implies $i(w) \geq \pm i(x)$, so $i(w) \geq|i(x)|=i(v)$, consequently $w \geq v$. Hence, $v=\sup \{x,-x\}=|x|$.

Corollary 36 Let $(X, K)$ be a pre-Riesz space and let $a, b \in K$. The following statements are equivalent.

(i) $a \perp b$.

(ii) The modulus of $a-b$ exists and equals $a+b$.

Proof Let $(Y, i)$ be a vector lattice cover of $X$.

(i) $\Rightarrow$ (ii): As $i(a)$ and $i(b)$ are positive and disjoint, we get

$$
|i(a-b)|=|i(a)-i(b)|=i(a)+i(b)=i(a+b) \in i[X] .
$$

By Theorem 35, $|a-b|$ exists in $X$. Lemma 34(a) implies that

$$
i(|a-b|)=|i(a-b)|=i(a+b),
$$

hence $|a-b|=a+b$.

(ii) $\Rightarrow$ (i): Using Lemma 34(a), we have

$$
|i(a)-i(b)|=|i(a-b)|=i(|a-b|)=i(a+b)=i(a)+i(b),
$$

hence $i(a) \wedge i(b)=\frac{1}{2}(i(a)+i(b)-|i(a)-i(b)|)=0$. We conclude that $a \perp b$.

Theorem 35 can be used to determine the set of elements that have a modulus in examples.

Example 37 (a) Let $V:=\mathbb{R}^{3}$ be equipped with the four-ray cone given in (11). The set $M \subseteq V$ of elements in $(V, K)$ that have a modulus is

$$
M=K \cup(-K) \cup E_{1} \cup E_{2},
$$

where $E_{1}=\left\{(0, u, v)^{T} ; u, v \in \mathbb{R}\right\}$ and $E_{2}=\left\{(u, 0, v)^{T} ; u, v \in \mathbb{R}\right\}$. Indeed, to apply Theorem 35, we need all pairs of (normed) nontrivial positive disjoint elements. (In other words, we need all directed bands.) In [9, Example 4.4.18], it is observed that there are only two such pairs, namely

$$
\left\{\left(\begin{array}{l}
0 \\
1 \\
1
\end{array}\right),\left(\begin{array}{c}
0 \\
-1 \\
1
\end{array}\right)\right\} \text { and }\left\{\left(\begin{array}{l}
1 \\
0 \\
1
\end{array}\right),\left(\begin{array}{c}
-1 \\
0 \\
1
\end{array}\right)\right\}
$$

Note that $E_{1}$ is the span of the first pair, and $E_{2}$ is the span of the second pair. 
(b) A generalization of (a) in $\mathbb{R}^{n}$ is given in [9, Example 1.7.7]. Consider the space $X:=\mathbb{R}^{n}(n \geq 3)$ with the following $(2 n-2)$-ray cone. Let $e^{(i)}$ denote the standard basis vectors, and for $i \in\{1, \ldots, n-1\}$, define $x^{(i)}:=e^{(i)}+e^{(n)}$ and $y^{(i)}:=-e^{(i)}+e^{(n)}$. Let

$$
K:=\operatorname{pos}\left\{x^{(1)}, \ldots, x^{(n-1)}, y^{(1)}, \ldots, y^{(n-1)}\right\}
$$

In [14, Example 4.2], all pairs of nontrivial disjoint elements are determined. The (normed) pairs in $K$ are $\left\{x^{(i)}, y^{(i)}\right\}$ for $i \in\{1, \ldots, n-1\}$. Hence, by Theorem 35 , the set $M \subseteq X$ of elements with modulus is

$$
M=K \cup(-K) \cup \bigcup_{i=1}^{n-1} \operatorname{span}\left\{x^{(i)}, y^{(i)}\right\}
$$

Remark 38 Note that (iv) in Theorem 35 is stronger than assuming that $D$ is a vector lattice. In Example 37(a), the subspace $D:=\left\{(u, u, v)^{T} ; u, v \in \mathbb{R}\right\}$ is a twodimensional vector lattice, but $i[D]$ is not a Riesz subspace in the Riesz completion.

It follows from Theorem 35 that an operator preserves moduli if and only if it is disjointness preserving on the positive elements, as we show next. Note that operators as in Proposition 39(ii) play a role in [6].

Proposition 39 Let $(X, K)$ and $V$ be pre-Riesz spaces, and let $T: X \rightarrow V$ be a positive linear map. Then the following statements are equivalent.

(i) For every $a, b \in K$ with $a \perp b$, we have $T a \perp T b$.

(ii) If $x \in X$ has a modulus, then Tx has a modulus in $V$, and $T|x|=|T x|$.

Proof (i) $\Rightarrow$ (ii): Let $(Y, i)$ be a vector lattice cover of $V$. Let $x \in X$ be such that $|x|$ exists. By Lemma 34(b), there exist $a, b \in K, a \perp b$, such that $x=a-b$. Hence, $i(T a) \perp i(T b)$, and $i(T a)$ and $i(T b)$ are positive. Therefore, by Corollary 36,

$$
\begin{aligned}
|i(T x)| & =|i(T(a-b))|=|i(T a)-i(T b)| \\
& =i(T a)+i(T b)=i(T(a+b))=i(T|x|) \in i[V] .
\end{aligned}
$$

By Theorem 35, $|T x|$ exists in $V$, and by Lemma 34(a), we get $i(|T x|)=|i(T x)|$. Now, (15) yields that $i(|T x|)=i(T|x|)$, which implies $|T x|=T|x|$.

(ii) $\Rightarrow$ (i): Let $a, b \in K$ be such that $a \perp b$. By Corollary 36, $|a-b|$ exists and equals $a+b$. By assumption, $|T(a-b)|$ exists and

$$
|T a-T b|=|T(a-b)|=T|a-b|=T a+T b .
$$

Again by Corollary 36, we obtain $T a \perp T b$.

Remark 40 Let $X$ and $Y$ be pre-Riesz spaces and $T: X \rightarrow Y$ a Riesz* homomorphism. Then $T$ is a positive disjointness preserving operator, hence also the condition in Proposition 39(ii) is satisfied. 
Note that Proposition 39 describes, in fact, the behaviour of operators on subspaces generated by two nontrivial positive disjoint elements.

Corollary 41 Let $X$ and $Y$ be pre-Riesz spaces and let $T: X \rightarrow Y$ be a positive linear map that is disjointness preserving on positive elements. Let $a$ and $b$ be nontrivial positive disjoint elements in $X$ and let $D:=\operatorname{span}\{a, b\}$. Then $T[D]$ is a Riesz space and $\left.T\right|_{D}: D \rightarrow T[D]$ is a Riesz homomorphism.

Proof By Corollary 36, $D$ is a Riesz space, and similarly, as $T(a) \perp T(b), T[D]$ is a Riesz space as well. According to Proposition 39, $\left.T\right|_{D}: D \rightarrow T[D]$ is a Riesz homomorphism.

As a consequence, in view of Remark 40, observe that Riesz* homomorphisms act as Riesz homomorphisms on two-dimensional subspaces spanned by two nontrivial positive disjoint elements.

\section{Relation between positive disjointness preserving operators and Riesz* homomorphisms}

For linear operators in Riesz spaces, it is well-known that an operator is positive and disjointness preserving if and only if it is a Riesz homomorphism. In pre-Riesz spaces, every Riesz* homomorphism is a positive disjointness preserving operator, see [9, Theorem 5.1.12]. We will deal with the converse implication and study the following question:

(Q) Let a positive linear map $T: X \rightarrow Y$ between pre-Riesz spaces be disjointness preserving on positive elements, as in Proposition 39. Under which conditions on $X$ and $Y$ do we have that $T$ is a Riesz* homomorphism?

Van Imhoff observed in [13, Example 2.29] that, in general, additional conditions are needed for $T$ to be a Riesz* homomorphism in (Q). The next example shows that even in the case of functionals extra conditions are necessary.

Example 42 Consider the Namioka space

$$
X=\{x \in \mathrm{C}[-1,1] ; 2 x(0)=x(1)+x(-1)\}
$$

with pointwise order and define the linear functional

$$
h: X \rightarrow \mathbb{R}, \quad x \mapsto 2 x(1)+x(-1)-x(0) .
$$

To show that $h$ is positive, let $x \in X, x \geq 0$. Then

$$
0 \leq x(0)=x(1)+x(-1)-x(0) \leq 2 x(1)+x(-1)-x(0)=h(x) .
$$

To prove that $h$ is disjointness preserving, let $x, y \in X$ with $x \perp y$. This implies that, for $q \in M:=\{1,-1,0\}$, we have $x(q)=0$ or $y(q)=0$. We need to show that 
$h(x)=0$ or $h(y)=0$. By symmetry, it is enough to consider the cases where $x$ equals zero for either none or exactly one of the elements of $M$. If $x(q) \neq 0$ for all $q \in M$, then $y(q)=0$ for all $q \in M$ and, therefore, $h(y)=0$. If there exists exactly one $q \in M$, say $q=1$, such that $x(1)=0$, then $y(-1)=y(0)=0$, and, by the definition of $X$, we get $y(1)=2 y(0)-y(-1)=0$ and therefore $h(y)=0$. The other cases are similar. Hence, $h$ is disjointness preserving.

Finally, we show that $h$ is not a Riesz* homomorphism. Suppose the contrary, then, by Theorem 12, there exist $\alpha>0$ and $t \in[-1,1]$ such that $h(x)=\alpha x(t)$ for every $x \in X$. Since $\mathbb{1}:[-1,1] \rightarrow \mathbb{R}, q \mapsto 1$, is in $X$, we get

$$
2=h(\mathbb{1})=\alpha \mathbb{1}(t)=\alpha .
$$

Next, consider the function $y \in X$ defined by $y(q):=\frac{1}{2} q+\frac{1}{2}, q \in[-1,1]$. Then

$$
\frac{3}{2}=h(y)=2 y(t)=2\left(\frac{1}{2} t+\frac{1}{2}\right)=t+1 .
$$

Consequently, $t=\frac{1}{2}$. Now, let $z \in X$ be given by

$$
z(q):= \begin{cases}2\left|q+\frac{1}{2}\right| & \text { if } q \in[-1,0] \\ 2\left|q-\frac{1}{2}\right| & \text { if } q \in(0,1] .\end{cases}
$$

Then we get $h(z)=2$ and $h(z)=2 z\left(\frac{1}{2}\right)=0$, a contradiction.

We study (Q) in spaces of continuous functions. For a nonempty compact Hausdorff space $P$ and $X \subseteq \mathrm{C}(P)$, we consider the condition

(SD) For every $p_{1}, p_{2} \in P$ with $p_{1} \neq p_{2}$, there exist $a_{1}, a_{2} \in X$ with $a_{1}, a_{2} \geq 0$ and $a_{1} \perp a_{2}$ such that $a_{1}\left(p_{1}\right) \neq 0$ and $a_{2}\left(p_{2}\right) \neq 0$,

i.e., the points of $P$ are separated by disjoint positive elements. Using the condition (SD), we obtain an affirmative answer to (Q) first for functionals, and then for operators in Theorem 49 below.

Proposition 43 Let $P$ be a nonempty compact Hausdorff space and let $X$ be an order dense subspace of $\mathrm{C}(P)$ such that $(S D)$ is satisfied. Then every positive linear functional $f: X \rightarrow \mathbb{R}$ which is disjointness preserving on positive elements is a Riesz* homomorphism.

Proof Let $f: X \rightarrow \mathbb{R}$ be a positive linear map which is disjointness preserving on positive elements. As $X$ is majorizing in $\mathrm{C}(P)$, there exists a positive linear map $\hat{f}: \mathrm{C}(P) \rightarrow \mathbb{R}$ extending $f$, due to the Kantorovich theorem [3, Theorem 1.36]. By the Riesz-Markov-Kakutani representation theorem, there exists a finite nonnegative regular Borel measure $\mu$ on $P$ such that $\hat{f}(x)=\int x \mathrm{~d} \mu$ for every $x \in \mathrm{C}(P)$. Consider the support of $\mu$ given by

$$
\operatorname{supp}(\mu):=\{p \in P ; \mu(U)>0 \text { for every open } U \subseteq P \text { with } p \in U\}
$$




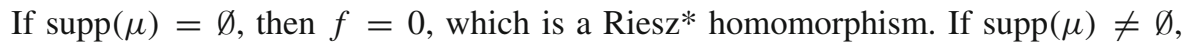
then we show that $\operatorname{supp}(\mu)$ consists of one point. Indeed, suppose there are $p_{1}, p_{2} \in$ $\operatorname{supp}(\mu)$ with $p_{1} \neq p_{2}$. By (SD), there exist positive disjoint elements $a_{1}, a_{2} \in X$ with $a_{1}\left(p_{1}\right)>0$ and $a_{2}\left(p_{2}\right)>0$. Choose $\varepsilon>0$ and an open set $U \subseteq P$ with $p_{1} \in U$ such that $a_{1}(p) \geq \varepsilon$ for every $p \in U$. Then

$$
f\left(a_{1}\right)=\hat{f}\left(a_{1}\right) \geq \int \varepsilon \mathbf{1}_{U} \mathrm{~d} \mu=\varepsilon \mu(U)>0,
$$

as $p_{1} \in \operatorname{supp}(\mu)$. Similarly, $f\left(a_{2}\right)>0$. Hence, $f\left(a_{1}\right)$ and $f\left(a_{2}\right)$ are not disjoint, which is a contradiction. Thus, there exists $p \in P$ such that $\operatorname{supp}(\mu)=\{p\}$. It follows that there exists $\omega>0$ such that $\mu=\omega \delta_{p}$, where $\delta_{p}$ is the point measure at $p$. Therefore, $\hat{f}(x)=\int x \mathrm{~d} \mu=\omega x(p)$ for every $x \in \mathrm{C}(P)$, which means that $\hat{f}$ is a Riesz homomorphism. As $X$ is order dense in $\mathrm{C}(P)$, we obtain that $f$ is a Riesz* homomorphism, due to Theorem 5 .

Example 44 For $X=\mathrm{C}^{1}[0,1] \subseteq \mathrm{C}[0,1]$, the condition (SD) is satisfied.

Example 45 Clearly, (SD) fails for every space $X \subseteq \mathrm{C}(P)$ that does not contain nonzero positive disjoint elements. By [9, Theorem 4.1.10], this means that (SD) is not satisfied whenever $X$ is an antilattice.

Example 46 In the Namioka space as in Example 42, the condition (SD) fails due to Proposition 43. To see this directly, take $p_{1}=-1$ and $p_{2}=1$. As $a_{1}, a_{2} \geq 0$ and $a_{1}\left(p_{1}\right)>0, a_{2}\left(p_{2}\right)>0$ implies that $a_{1}(0)>0$ and $a_{2}(0)>0$, we obtain that $a_{1}$ and $a_{2}$ are not disjoint.

Observe that the sufficient condition (SD) in Proposition 43 is not a necessary one, as the next example shows.

Example 47 Let $X:=\{x \in \mathrm{C}[0,1] ; x(0)=x(1)\}$. On the one hand, as $X$ is a Riesz space, every positive disjointness preserving functional is a Riesz homomorphism, and, hence, a Riesz* homomorphism. On the other hand, (SD) does not hold. Indeed, let $p_{1}=0, p_{2}=1$ and let $a_{1}, a_{2} \geq 0$ be disjoint elements in $X$ such that $a_{1}(0) \neq 0$. Then $a_{2}(0)=0$, which implies $a_{2}(1)=0$. Note that $X$ is isomorphic to $\mathrm{C}(\mathrm{S})$, where $\mathrm{S}$ denotes the unit circle in $\mathbb{R}^{2}$. Observe that $\mathrm{C}(\mathrm{S})$ satisfies $(\mathrm{SD})$.

If (SD) is satisfied for $X \subseteq \mathrm{C}(P)$, then, in particular, $a_{1}\left(p_{2}\right)=0$, hence the following is obvious.

Lemma 48 If $X \subseteq \mathrm{C}(P)$ satisfies $(S D)$, then $X$ strongly separates the points of $P$.

The next result provides an answer to question (Q) for pre-Riesz spaces consisting of continuous functions.

Theorem 49 Let $P$ and $Q$ be nonempty compact Hausdorff spaces, let $X$ be an order dense subspace of $\mathrm{C}(P)$ that satisfies $(S D)$, and let $Y$ be an order dense subspace of $\mathrm{C}(Q)$. Then every positive linear map $T: X \rightarrow Y$ which is disjointness preserving on the positive elements of $X$ is a Riesz* homomorphism. 
Proof Let $q \in Q$. Then $f_{q}: X \rightarrow \mathbb{R}, x \mapsto(T x)(q)$, is a positive linear map which is disjointness preserving on positive elements of $X$. By Proposition 43, the functional $f_{q}$ is a Riesz* homomorphism. We extend $f_{q}$ first to a Riesz homomorphism on the Riesz completion of $X$ by means of Theorem 5, and then to a Riesz homomorphism $\hat{f}_{q}: \mathrm{C}(P) \rightarrow \mathbb{R}$, using the Lipecki-Luxemburg-Schep theorem, see, e.g., [9, Theorem 2.1.17]. Now there exist $w(q) \in \mathbb{R}_{+}$and $\alpha(q) \in P$ such that $\hat{f}_{q}(x)=w(q) x(\alpha(q))$ for every $x \in \mathrm{C}(P)$, see, e.g., [9, Theorem 5.1.13]. Moreover, for every $x \in X$, we obtain

$$
(T x)(q)=f_{q}(x)=\hat{f}_{q}(x)=w(q) x(\alpha(q)) .
$$

By Lemma 48, the space $X$ strongly separates the points of $P$. Next we apply Proposition 13. As $T x \in \mathrm{C}(Q)$ for every $x \in X$, we get that $w \in \mathrm{C}(Q)$ and that $\alpha$ is continuous on $\{q \in Q ; w(q)>0\}$. Now it follows from Theorem 12(ii) that $T: X \rightarrow Y$ is a Riesz* homomorphism.

A result similar to Theorem 49 was shown by van Imhoff under the additional assumption that $X$ and $Y$ are pointwise pervasive, see [13, Theorem 2.30]. We show that his result is a special case of Theorem 49. For a nonempty compact Hausdorff space $P$, a set $X \subseteq \mathrm{C}(P)$ is called pointwise pervasive if for every $p \in P$ and every neighbourhood $U \subseteq P$ of $p$, there exists $x \in X, x \geq 0$, such that $x(p) \neq 0$ and $\operatorname{coz}(x) \subseteq U$. If $X$ is pointwise pervasive, then $X$ is pervasive, see Proposition 16 .

Proposition 50 Let $P$ be a nonempty Hausdorff space and let $X \subseteq \mathrm{C}(P)$ be pointwise pervasive. Then $X$ satisfies $(S D)$.

Proof Let $p_{1}, p_{2} \in P, p_{1} \neq p_{2}$. Since $P$ is Hausdorff, there exist disjoint neighbourhoods $V_{1}, V_{2}$ of $p_{1}, p_{2}$, respectively. As $X$ is pointwise pervasive, there are $x_{1}, x_{2} \in X$, $x_{1}, x_{2} \geq 0$, such that $x_{i}\left(p_{i}\right) \neq 0$ and $\operatorname{coz}\left(x_{i}\right) \subseteq V_{i}$ for $i \in\{1,2\}$. The disjointness of $V_{1}$ and $V_{2}$ implies that $x_{1} \perp x_{2}$.

We reformulate Theorem 49 for order unit spaces, where we use the functional representation.

Corollary 51 For an order unit space $\left(X, K,\|\cdot\|_{u}\right)$, consider the following three statements.

(a) For every Riesz* homomorphisms $\varphi_{1}, \varphi_{2}: X \rightarrow \mathbb{R}$ with $\varphi_{1}(u)=\varphi_{2}(u)=1$ and $\varphi_{1} \neq \varphi_{2}$, there exist disjoint elements $a_{1}, a_{2} \in K$ such that $\varphi_{1}\left(a_{1}\right) \neq 0$ and $\varphi_{2}\left(a_{2}\right) \neq 0$.

(b) For the functional representation $(\Phi, \bar{\Lambda})$ of $\left(X, K,\|\cdot\|_{u}\right)$ given in (5), we have that $\Phi[X] \subseteq \mathrm{C}(\bar{\Lambda})$ satisfies $(S D)$.

(c) For every order unit space $\left(Y, C,\|\cdot\|_{v}\right)$ and every positive linear map $T: X \rightarrow Y$ that is disjointness preserving on $K$, we have that $T$ is a Riesz* homomorphism.

Then (a) and (b) are equivalent, and (b) implies (c).

Proof $(\mathrm{a}) \Rightarrow(\mathrm{b})$ : Let $p_{1}, p_{2} \in \bar{\Lambda}$ be such that $p_{1} \neq p_{2}$. Then $p_{1}, p_{2}$ are Riesz* homomorphisms due to Proposition 17 , and $p_{1}(u)=p_{2}(u)=1$. By assumption, 
there exist disjoint elements $a_{1}, a_{2} \in K$ such that $p_{1}\left(a_{1}\right) \neq 0$ and $p_{2}\left(a_{2}\right) \neq 0$. Then $\Phi\left(a_{1}\right)$ and $\Phi\left(a_{2}\right)$ are positive disjoint elements in $\Phi[X]$ with $\Phi\left(a_{1}\right)\left(p_{1}\right)=p_{1}\left(a_{1}\right) \neq 0$ and $\Phi\left(a_{2}\right)\left(p_{2}\right)=p_{2}\left(a_{2}\right) \neq 0$.

(b) $\Rightarrow$ (a): Let $\varphi_{1}, \varphi_{2} \in \bar{\Lambda}, \varphi_{1} \neq \varphi_{2}$. As $\Phi[X]$ satisfies (SD), there are $b_{1}, b_{2} \in \Phi[X]$ with $b_{1}, b_{2} \geq 0$ and $b_{1} \perp b_{2}$ such that $b_{1}\left(\varphi_{1}\right) \neq 0$ and $b_{2}\left(\varphi_{2}\right) \neq 0$. There are $a_{1}, a_{2} \in X$ with $b_{1}=\Phi\left(a_{1}\right), b_{2}=\Phi\left(a_{2}\right)$. We get $a_{1}, a_{2} \geq 0, a_{1} \perp a_{2}, \varphi_{1}\left(a_{1}\right)=$ $\Phi\left(a_{1}\right)\left(\varphi_{1}\right) \neq 0$ and $\varphi_{2}\left(a_{2}\right)=\Phi\left(a_{2}\right)\left(\varphi_{2}\right) \neq 0$.

(b) $\Rightarrow$ (c): As $X$ and $\Phi[X]$ are isomorphic as partially ordered vector spaces, we apply Theorem 49, where, by means of the functional representation, we also consider $Y$ as an order dense subspace of an appropriate space of continuous functions.

Corollary 51 is convenient for the study of examples. In the subsequent one, we furthermore show that, in general, (SD) is a weaker condition than being pointwise pervasive.

Example 52 For the four-ray cone in Example 37(a), the statement (a) in Corollary 51 holds. We continue Example 37(b), i.e., we study the $(2 n-2)$-ray cone $K$ in $X:=\mathbb{R}^{n}$ further. The element $u:=e^{(n)}$ is an order unit in $X$ and, by [14, Example 4.1], we get in (5) that

$$
\Lambda=\left\{f_{\sigma} \in K^{\prime} ; \sigma \in\{-1,1\}^{n-1}\right\}
$$

where $f_{\sigma}(x):=\sum_{i=1}^{n-1} \sigma_{i} x_{i}+x_{n}$. In [14, Section 4], all positive disjointness preserving bijections $T: X \rightarrow X$ are calculated. We show now that every positive linear map that is disjointness preserving on $K$ is a complete Riesz homomorphism. First, we establish that (a) in Corollary 51 is satisfied. Indeed, let $f_{\sigma}, f_{\delta} \in \Lambda\left(\sigma, \delta \in\{-1,1\}^{n-1}\right)$ with $f_{\sigma} \neq f_{\delta}$. This implies that $\sigma \neq \delta$. Therefore, there exists $j \in\{1, \ldots, n-1\}$ such that $\sigma_{j} \neq \delta_{j}$, say $\sigma_{j}=1, \delta_{j}=-1$. We get $f_{\sigma}\left(x^{(j)}\right)=\sigma_{j}+1=2 \neq 0$ and $f_{\delta}\left(y^{(j)}\right)=-\delta_{j}+1=2 \neq 0$. Since $x^{(j)}, y^{(j)} \geq 0$ and $x^{(j)} \perp y^{(j)}$, we have shown that $X$ satisfies Corollary 51(a), which implies that $\Phi[X]$ has the property (SD) in $\mathrm{C}(\Lambda)$. Now, let $Y$ be an order unit space and let $T: X \rightarrow Y$ be a positive linear map that is disjointness preserving on $K$. So far, we have shown that $T$ is a Riesz* homomorphism. By Corollary 31, the map $T$ is a complete Riesz homomorphism.

We show that $X$ is not pervasive and, therefore, not pointwise pervasive. Indeed, using (5) and (16), we obtain $\Phi: X \rightarrow \mathbb{R}^{2^{n-1}}$ given by

$$
\Phi(x)=\sum_{j=1}^{2^{n-1}} f_{\gamma(j)}(x) e^{(j)}, \quad x \in X
$$

where $\gamma:\left\{1, \ldots, 2^{n-1}\right\} \rightarrow\left\{\sigma ; \sigma \in\{-1,1\}^{n-1}\right\}$ is a bijection. We get $\left(\mathbb{R}^{2^{n-1}}, \Phi\right)$ as the functional representation of $X$. In view of [9, Proposition 4.1.15 and Theorem 4.2.6], we show that there exists a band $B$ in $\mathbb{R}^{2^{n-1}}$ such that $\{x \in X ; \Phi(x) \in B\}$ is not a band in $X$. Let

$$
B:=\operatorname{span}\left\{e^{(i)} ; i \in\left\{2, \ldots, 2^{n-1}\right\}\right\} \subseteq \mathbb{R}^{2^{n-1}}
$$


which is a band, see, e.g., [9, Example 1.3.14]. Denote $I_{+}:=\{i \in\{1, \ldots, n-$ $\left.1\} ; \gamma(1)_{i}=1\right\}$ and $I_{-}:=\left\{i \in\{1, \ldots, n-1\} ; \gamma(1)_{i}=-1\right\}$. We get

$$
\begin{aligned}
\{x \in X ; \Phi(x) \in B\} & =\left\{x \in X ; f_{\gamma(1)}=0\right\} \\
& \supseteq \operatorname{span}\left(\left\{x^{(i)} ; i \in I_{-}\right\} \cup\left\{y^{(i)} ; i \in I_{+}\right\}\right),
\end{aligned}
$$

where the latter is at least two-dimensional, as $n \geq 3$. In [14, Example 4.2], it is shown that every band in $X$ equals either span $\left\{e^{(i)}+e^{(j)}\right\}$ or span $\left\{e^{(i)}-e^{(j)}\right\}$ for some $i, j \in \mathbb{N}, 1 \leq i<j \leq n$. Hence, $\{x \in X ; \Phi(x) \in B\}$ is not a band. We conclude that $X$ is not pervasive.

Next, we discuss to what extent the statement in Corollary 51(b) is necessary for the one in (c).

Proposition 53 Let $\left(X, K,\|\cdot\|_{u}\right)$ be an order unit spaces and $(\mathrm{C}(\bar{\Lambda}), \Phi)$ the functional representation of $X$. Then the following statements are equivalent.

(i) $\Phi[X] \subseteq \mathrm{C}(\bar{\Lambda})$ satisfies $(S D)$.

(ii) For every $\varphi_{1}, \varphi_{2} \in \bar{\Lambda}, \varphi_{1} \neq \varphi_{2}$, we have that $\varphi_{1}+\varphi_{2}$ is not disjointness preserving on $K$.

(iii) For every $\varphi \in \Sigma$ that is disjointness preserving on $K$, we have that $\varphi \in \bar{\Lambda}$, and for every $\varphi_{1}, \varphi_{2} \in \bar{\Lambda}, \varphi_{1} \neq \varphi_{2}$, we have that $\frac{1}{2}\left(\varphi_{1}+\varphi_{2}\right) \notin \bar{\Lambda}$.

(iv) For every order unit space $\left(Y, C,\|\cdot\|_{v}\right)$, we have that every positive linear map $T: X \rightarrow Y$ that is disjointness preserving on $K$ is a Riesz* homomorphism, and for every $\varphi_{1}, \varphi_{2} \in \bar{\Lambda}, \varphi_{1} \neq \varphi_{2}$, we have that $\frac{1}{2}\left(\varphi_{1}+\varphi_{2}\right) \notin \bar{\Lambda}$.

Proof (i) $\Rightarrow$ (ii): Let $\varphi_{1}, \varphi_{2} \in \bar{\Lambda}, \varphi_{1} \neq \varphi_{2}$, and define $\varphi:=\varphi_{1}+\varphi_{2}$. Since $\Phi[X]$ satisfies (SD), there exist disjoint elements $a, b \in K$ such that $\varphi_{1}(a)>0, \varphi_{2}(b)>0$. By the positivity of $\varphi_{1}, \varphi_{2}$, we also have $\varphi_{1}(b) \geq 0, \varphi_{2}(a) \geq 0$. Hence, we get

$$
\varphi(a)=\varphi_{1}(a)+\varphi_{2}(a)>0, \quad \varphi(b)=\varphi_{1}(b)+\varphi_{2}(b)>0 .
$$

Therefore, $\varphi$ is not disjointness preserving on $K$.

(ii) $\Rightarrow$ (i): Assume that $\Phi[X]$ does not have the property (SD). By Corollary 51, there exist $\varphi_{1}, \varphi_{2} \in \bar{\Lambda}, \varphi_{1} \neq \varphi_{2}$, such that for every $a, b \in K$ with $a \perp b$, we have

$$
\varphi_{1}(a) \neq 0 \Rightarrow \varphi_{2}(b)=0 \text { and } \varphi_{1}(b) \neq 0 \Rightarrow \varphi_{2}(a)=0 .
$$

We show that $\varphi:=\varphi_{1}+\varphi_{2}$ is disjointness preserving on $K$. Let $a, b \in K$ be disjoint. Then, since $\varphi_{1}, \varphi_{2}$ are disjointness preserving, $\varphi_{1}(a) \varphi_{1}(b)=\varphi_{2}(a) \varphi_{2}(b)=0$. We get

$$
\begin{aligned}
\varphi(a) \varphi(b) & =\varphi_{1}(a) \varphi_{1}(b)+\varphi_{1}(a) \varphi_{2}(b)+\varphi_{2}(a) \varphi_{1}(b)+\varphi_{2}(a) \varphi_{2}(b) \\
& =\varphi_{1}(a) \varphi_{2}(b)+\varphi_{2}(a) \varphi_{1}(b) .
\end{aligned}
$$

If $\varphi_{1}(a) \neq 0$, then we have $\varphi_{1}(b)=0$ by the disjointness of $a$ and $b$, and $\varphi_{2}(b)=0$ by (17), hence $\varphi(a) \varphi(b)=0$. If $\varphi_{1}(a)=0$ and $\varphi_{1}(b)=0$, we also have $\varphi(a) \varphi(b)=0$. If 
$\varphi_{1}(a)=0$ and $\varphi_{1}(b) \neq 0$, then we have by (17) that $\varphi_{2}(a)=0$, hence $\varphi(a) \varphi(b)=0$. We conclude that $\varphi$ is disjointness preserving on $K$.

(ii) $\Rightarrow$ (iv): As (ii) implies (i), Corollary 51 yields that that every positive linear map $T: X \rightarrow Y$ that is disjointness preserving on $K$ is a Riesz* homomorphism. Furthermore, for every $\varphi_{1}, \varphi_{2} \in \bar{\Lambda}, \varphi_{1} \neq \varphi_{2}$, we have that $\varphi_{1}+\varphi_{2}$ is not disjointness preserving on $K$, hence not a Riesz $*$ homomorphism. Therefore, $\frac{1}{2}\left(\varphi_{1}+\varphi_{2}\right) \notin \bar{\Lambda}$. (iv) $\Rightarrow$ (iii) is obvious.

(iii) $\Rightarrow$ (ii): Let $\varphi_{1}, \varphi_{2} \in \bar{\Lambda}, \varphi_{1} \neq \varphi_{2}$. Assume that $\varphi:=\varphi_{1}+\varphi_{2}$ is disjointness preserving on $K$. Then $\frac{1}{2} \varphi$ is also disjointness preserving on $K$ and therefore, by assumption, a Riesz* homomorphism. Hence $\frac{1}{2} \varphi \in \bar{\Lambda}$.

\section{Operators with modulus}

In this section, we apply the results of Section 6 to obtain moduli of functionals and operators. We start by recalling the four-ray cone example and characterize functionals that have a modulus.

Example 54 We change the point of view in Example 37(a), where in $V=\mathbb{R}^{3}$ the four-ray cone $K$, see (11), is considered. Let the space $X:=\mathbb{R}^{3}$ be equipped with the cone

$$
C:=\operatorname{pos}\left\{f^{(1)}, f^{(2)}, f^{(3)}, f^{(4)}\right\}
$$

using (12). Then, by duality, $\mathrm{L}^{\mathrm{b}}(X, \mathbb{R})$ can be identified with $(V, K)$. We already calculated the set $M$ of functionals in $\mathrm{L}^{\mathrm{b}}(X, \mathbb{R})$ that have a modulus, see (14).

The method in the previous example is a special case of the subsequent Proposition 56 on finite-dimensional order unit spaces. Let $K$ be a closed generating cone in $\mathbb{R}^{n}$. Then $K$ contains an order unit $u$, and the dual cone $K^{\prime}:=\left\{y \in \mathbb{R}^{n} ; \forall x \in\right.$ $K:\langle x, y\rangle \geq 0\}$ is generating in $X^{\prime}$. As $K^{\prime}$ is closed, it follows that $\left(\mathbb{R}^{n}, K^{\prime}\right)$ is also an order unit space. For a fixed order unit $v \in K^{\prime}$, we consider the functional representation $(\mathrm{C}(\bar{L}), \Phi)$ of $\left(\mathbb{R}^{n}, K^{\prime},\|\cdot\|_{v}\right)$, see (5). Note that $\bar{L} \subseteq K^{\prime \prime}=K$ (see also [9, Lemma 2.6.8]), that $L$ consists of the extreme points of the base $S=\{x \in K ;\langle x, v\rangle=1\}$ of $K$, and that $\bar{L}$ can be considered as the closure of $L$ in $S$. The next lemma states that two functionals on $\left(\mathbb{R}^{n}, K\right)$ are disjoint if and only if they are pointwise disjoint on $\bar{L}$.

Lemma 55 For every $a, b \in K^{\prime} \backslash\{0\}$, we have $a \perp b$ in $\left(\mathbb{R}^{n}, K^{\prime}\right)$ if and only if, for every $x \in \bar{L}$, we have $a(x)=0$ or $b(x)=0$.

Proof Since $(\mathrm{C}(\bar{L}), \Phi)$ is a vector lattice cover of $\left(\mathbb{R}^{n}, K^{\prime}\right)$, we have, for every $a, b \in$ $\mathbb{R}^{n}$, that $a \perp b$ in $\left(\mathbb{R}^{n}, K^{\prime}\right)$ if and only if $\Phi(a) \perp \Phi(b)$ in $\mathrm{C}(\bar{L})$, where the latter means that, for every $x \in \bar{L}$, we have $a(x)=0$ or $b(x)=0$.

Now, we characterize functionals that have a modulus. The subsequent statement is a direct consequence of Theorem 35 and Lemma 55. 
Proposition 56 Let $K$ be a closed generating cone in $\mathbb{R}^{n}$, let $v$ be an order unit in $K^{\prime}$, and let $(\mathrm{C}(\bar{L}), \Phi)$ be the functional representation of $\left(\mathbb{R}^{n}, K^{\prime},\|\cdot\|_{v}\right)$. Let $f: \mathbb{R}^{n} \rightarrow \mathbb{R}$ be a linear functional. Then the following are equivalent.

(i) $f$ has a modulus in $\left(\mathbb{R}^{n}, K^{\prime}\right)$.

(ii) Either $f \in K^{\prime} \cup\left(-K^{\prime}\right)$, or there exist $a, b \in K^{\prime} \backslash\{0\}$ with $f \in \operatorname{span}\{a, b\}$ such that, for every $x \in \bar{L}$, one has $a(x)=0$ or $b(x)=0$.

If a functional as in Proposition 56 has a modulus, then the modulus is given by the Riesz-Kantorovich formula due to [4, Theorem 3.3]. Proposition 56(ii) can also be replaced by

(ii') either $f \in K^{\prime} \cup\left(-K^{\prime}\right)$, or there exist $a, b \in \partial \Sigma$ with $f \in \operatorname{span}\{a, b\}$ such that for every $x \in \bar{L}$ one has $a(x)=0$ or $b(x)=0$,

where $\Sigma$ is given in (4). Indeed, by Remark 21, for $a, b \in K^{\prime} \backslash\{0\}, a \perp b$ can only occur if $a$ and $b$ are elements of the boundary of $K^{\prime}$, and $a$ and $b$ can then be scaled appropriately.

Example 57 We continue Example 37(b), i.e., we consider the $(2 n-2)$-ray cone in $X:=\mathbb{R}^{n}$ where, for the order unit $v: X \rightarrow \mathbb{R}, x \mapsto x_{n}$, in $K^{\prime}$, one has

$$
\bar{L}=L=\left\{x^{(1)}, \ldots, x^{(n-1)}, y^{(1)}, \ldots, y^{(n-1)}\right\} .
$$

We calculate all pairs $\{a, b\}$ of disjoint elements in $\partial \Sigma$, where the latter means that the $n$th entry of $a$ and $b$ equals 1 . By Lemma 55, two disjoint elements are pointwise disjoint on $L$. If, for $i \in\{1, \ldots, n-1\}$, we have $a_{i}+1=a\left(x^{(i)}\right)=0$, it follows $a\left(y^{(i)}\right)=-a_{i}+1=2 \neq 0$, hence $-b_{i}+1=b\left(y^{(i)}\right)=0$. We get $\{a, b\} \subseteq \Lambda$, and if $a=f_{\sigma}$, then $b=f_{\tilde{\sigma}}$, where, for $j \in\{1, \ldots, n-1\}$, we define $\tilde{\sigma}_{j}:=-\sigma_{j}$. By Proposition 56, the set $M$ of elements in $X^{\prime}$ that have a modulus is

$$
M:=K^{\prime} \cup\left(-K^{\prime}\right) \cup \bigcup_{\sigma \in\{-1,1\}^{n-1}} \operatorname{span}\left\{f_{\sigma}, f_{\tilde{\sigma}}\right\}
$$

Remark 58 Proposition 56(ii) can be reformulated by means of bands as follows,

(ii”) either $f \in K^{\prime} \cup\left(-K^{\prime}\right)$, or there exist a band $B$ in $X^{\prime}, a \in B \cap\left(K^{\prime} \backslash\{0\}\right)$, $b \in B^{\mathrm{d}} \cap\left(K^{\prime} \backslash\{0\}\right)$ such that $f \in \operatorname{span}\{a, b\}$.

Indeed, if $a \perp b$, then, for $B:=\{a\}^{\mathrm{dd}}$, we get $b \in\{a\}^{\mathrm{d}}=B^{\mathrm{d}}$. Note that bands in $X^{\prime}$ are determined by bisaturated subsets of $\bar{L}$, see [9, Theorem 4.4.17].

In the subsequent example, we use a vector lattice cover of a space of operators on a Riesz space to find the operators with modulus. Note that the underlying Riesz space is not Dedekind complete, so that we can not apply the Riesz-Kantorovich theory.

Example 59 Let $\ell_{0}^{\infty}$ denote the space of all finally constant real sequences, i.e.,

$$
\ell_{0}^{\infty}:=\left\{\left(x_{i}\right)_{i \in \mathbb{N}} ; \exists \beta \in \mathbb{R} \exists k \in \mathbb{N} \forall i>k: x_{i}=\beta\right\},
$$


equipped with the coordinatewise order. This vector lattice is not Dedekind complete. In [1, Theorem 4.1], it is established that $\mathrm{L}^{\mathrm{r}}\left(\ell_{0}^{\infty}\right)=\mathrm{L}^{\mathrm{b}}\left(\ell_{0}^{\infty}\right)$, and in [1, Theorem 5.1] that $\mathrm{L}^{\mathrm{r}}\left(\ell_{0}^{\infty}\right)$ does not satisfy the Riesz decomposition property, so it is not a vector lattice. In [7, Example 3.1], it is shown that $\mathrm{L}^{\mathrm{r}}\left(\ell_{0}^{\infty}\right)$ is a pervasive pre-Riesz space, and a vector lattice cover is calculated explicitely, where the order in the vector lattice cover is pointwise. For details, see also [9, Example 5.4.15].

More precisely, observe that $\ell_{0}^{\infty}$ has a countable algebraic basis $\mathcal{B}=\left\{\mathbb{1}, e^{(1)}, e^{(2)}\right.$, $\ldots\}$, where $\mathbb{1}$ denotes the constant- 1 sequence and $e^{(i)}$ the unit sequences. Hence, $\ell_{0}^{\infty}$ can be identified with the space $c_{c}$ of all real sequences which are zero except for a finite number of terms, ordered by the cone

$$
K=\left\{(\xi)_{i \in \mathbb{N}_{0}} \in \mathrm{c}_{\mathrm{c}} ; \xi_{0} \geq 0, \xi_{i}+\xi_{0} \geq 0 \text { for every } i \in \mathbb{N}\right\}
$$

The space $\mathrm{L}^{\mathrm{r}}\left(\ell_{0}^{\infty}\right)$ can be identified with the space of matrix representations of regular operators in $\left(\mathrm{c}_{\mathrm{c}}, K\right)$, i.e.,

$$
\mathcal{R}=\left\{\left(a_{i j}\right)_{i, j \in \mathbb{N}_{0}} ; \forall j \in \mathbb{N}_{0}:\left(a_{i j}\right)_{i} \in \mathrm{c}_{\mathrm{c}} \text {, and }\left(\sum_{j=1}^{\infty}\left|a_{i j}\right|\right)_{i \in \mathbb{N}_{0}} \text { is bounded }\right\}
$$

ordered by the cone

$$
\begin{aligned}
& \mathcal{C}=\left\{\left(a_{i j}\right)_{i, j \in \mathbb{N}_{0}} \in \mathcal{R} ; \forall i, j \in \mathbb{N}: a_{0 j}+a_{i j} \geq 0,\right. \\
& \text { and } \left.\forall i \in \mathbb{N}: a_{00}+a_{i 0} \geq \sum_{j=1}^{\infty}\left(a_{0 j}+a_{i j}\right)\right\} \text {. }
\end{aligned}
$$

The pre-Riesz space $(\mathcal{R}, \mathcal{C})$ can be embedded order densely into the vector lattice $Y$ of all matrices $\left(b_{i j}\right)_{i \in \mathbb{N}, j \in \mathbb{N}_{0}}$ that satisfy the four conditions

$$
\begin{aligned}
& \left(b_{i j}\right)_{i \in \mathbb{N}} \text { is eventually constant for every } j \geq 1, \\
& \sum_{j=1}^{\infty}\left|\beta_{j}\right|<\infty, \text { where } \beta_{j}=\lim _{i \rightarrow \infty} b_{i j} \\
& \left(b_{i 0}\right)_{i \in \mathbb{N}} \text { is bounded, } \\
& \left(\sum_{j=1}^{\infty}\left|b_{i j}\right|\right)_{i \in \mathbb{N}} \text { is bounded, }
\end{aligned}
$$

where $Y$ is endowed with the entrywise order. Observe that $Y$ is not $\sigma$-Dedekind complete. The map $F: \mathcal{R} \rightarrow Y$ given by

$$
A=\left(a_{i j}\right)_{i, j \in \mathbb{N}_{0}} \mapsto F(A)=\left(f_{i j}(A)\right)_{i \in \mathbb{N}, j \in \mathbb{N}_{0}},
$$


where

$$
f_{i j}(A)=\left\{\begin{array}{cl}
a_{0 j}+a_{i j} & \text { for } i \in \mathbb{N}, \quad j \geq 1, \\
a_{00}+a_{i 0}-\sum_{\ell=1}^{\infty}\left(a_{0 \ell}+a_{i \ell}\right) & \text { for } i \in \mathbb{N}, \quad j=0,
\end{array}\right.
$$

is bipositive, linear, and $F[\mathcal{R}]$ is order dense in $Y$. We obtain that $(Y, F)$ is a vector lattice cover of $(\mathcal{R}, \mathcal{C})$.

Whether an element of $\mathcal{R}$ has a modulus can be decided by checking if a certain sequence is finally constant. For a fixed $A=\left(a_{i j}\right)_{i, j \in \mathbb{N}_{0}} \in \mathcal{R}$, denote, for every $i \in \mathbb{N}$,

$$
s_{i}:=\left|a_{00}-\sum_{l=1}^{\infty}\left(a_{0 l}+a_{i l}\right)\right|+\sum_{l=1}^{\infty}\left|a_{0 l}+a_{i l}\right| .
$$

We claim that $A$ has a modulus $|A| \in \mathcal{R}$ if and only if $\left(s_{i}\right)_{i \in \mathbb{N}} \in \ell_{0}^{\infty}$. Applying Theorem 35, we show that there is $E=\left(e_{i j}\right)_{i, j \in \mathbb{N}_{0}} \in \mathcal{R}$ such that $|\mathcal{F}(A)|=\mathcal{F}(E)$ if and only if $\left(s_{i}\right)_{i \in \mathbb{N}} \in \ell_{0}^{\infty}$.

Indeed, assuming that $E=\left(e_{i j}\right)_{i, j \in \mathbb{N}_{0}} \in \mathcal{R}$ exists with $|\mathcal{F}(A)|=\mathcal{F}(E)$, one obtains

$$
\begin{aligned}
& e_{0 j}+e_{i j}=\left|a_{0 j}+a_{i j}\right| \quad \text { for } i, j \in \mathbb{N}, \\
& e_{00}+e_{i 0}-\sum_{l=1}^{\infty}\left(e_{0 l}+e_{i l}\right)=\left|a_{00}+a_{i 0}-\sum_{l=1}^{\infty}\left(a_{0 l}+a_{i l}\right)\right| \quad \text { for } i \in \mathbb{N} .
\end{aligned}
$$

Since $\left(a_{i j}\right)_{i \in \mathbb{N}_{0}} \in \mathrm{c}_{\mathrm{c}}$ and $\left(e_{i j}\right)_{i \in \mathbb{N}_{0}} \in \mathrm{c}_{\mathrm{c}},(18)$ implies $e_{0 j}=\left|a_{0 j}\right|$ for $j \in \mathbb{N}$ and, moreover, $e_{i j}=\left|a_{0 j}+a_{i j}\right|-\left|a_{0 j}\right|$ for $i, j \in \mathbb{N}$. Now (19) yields

$$
e_{00}+e_{i 0}=\left|a_{00}+a_{i 0}-\sum_{l=1}^{\infty}\left(a_{0 l}+a_{i l}\right)\right|+\sum_{l=1}^{\infty}\left|a_{0 l}+a_{i l}\right| .
$$

Since $\left(a_{i 0}\right)_{i \in \mathbb{N}_{0}} \in \mathrm{c}_{\mathrm{c}}$ and $\left(e_{i 0}\right)_{i \in \mathbb{N}_{0}} \in \mathrm{c}_{\mathrm{c}}$, it follows that $\left(s_{i}\right)_{i \in \mathbb{N}}$ is finally constant, where the limit equals $e_{00}$.

Vice versa, let the sequence $\left(s_{i}\right)_{i \in \mathbb{N}}$ be finally constant. Define $E:=\left(e_{i j}\right)_{i, j \in \mathbb{N}_{0}}$ by

$$
\begin{aligned}
& e_{00}=\lim _{i \rightarrow \infty} s_{i}, \\
& e_{i 0}=\left|a_{00}+a_{i 0}-\sum_{l=1}^{\infty}\left(a_{0 l}+a_{i l}\right)\right|+\sum_{l=1}^{\infty}\left|a_{0 l}+a_{i l}\right|-e_{00} \quad \text { for } i \in \mathbb{N}, \\
& e_{0 j}=\left|a_{0 j}\right| \quad \text { for } j \in \mathbb{N}, \\
& e_{i j}=\left|a_{0 j}+a_{i j}\right|-\left|a_{0 j}\right| \quad \text { for } i, j \in \mathbb{N} .
\end{aligned}
$$

We show that $E \in \mathcal{R}$. Since $\left(a_{i 0}\right)_{i \in \mathbb{N}}$ is finally constant and $\left(s_{i}\right)_{i \in \mathbb{N}_{0}}$ is finally constant with limit $e_{00}$, we obtain $\left(e_{i 0}\right)_{i \in \mathbb{N}_{0}} \in \mathrm{c}_{\mathrm{c}}$. As $\left(a_{i j}\right)_{i \in \mathbb{N}_{0}} \in \mathrm{c}_{\mathrm{c}}$ for every $j \in \mathbb{N}$, it follows 
that $\left(e_{i j}\right)_{i \in \mathbb{N}_{0}} \in \mathrm{c}_{\mathrm{c}}$, as well. Finally, since there is $M \geq 0$ such that $\sum_{j=1}^{\infty}\left|a_{i j}\right| \leq M$ for every $i \in \mathbb{N}_{0}$, we obtain

$$
\sum_{j=1}^{\infty}\left|e_{i j}\right|=\sum_{j=1}^{\infty}|| a_{0 j}+a_{i j}|-| a_{0 j}|| \leq 3 M
$$

hence the sequence $\left(\sum_{j=1}^{\infty}\left|e_{i j}\right|\right)_{i \in \mathbb{N}_{0}}$ is bounded. We conclude $E \in \mathcal{R}$. The verification of $\mathcal{F}(E)=|\mathcal{F}(A)|$ is straightforward.

Next, we calculate the modulus of certain operators between spaces of continuous functions.

Proposition 60 Let $P$ and $Q$ be nonempty compact Hausdorff spaces and let $X \subseteq$ $\mathrm{C}(P)$ and $Y \subseteq \mathrm{C}(Q)$ be directed subspaces. Let $\alpha: Q \rightarrow P$ and $w: Q \rightarrow \mathbb{R}$ and consider $T: \mathrm{C}(P) \rightarrow \mathbb{R}^{Q}$,

$$
(T x)(q):=w(q) x(\alpha(q)), \quad q \in Q, x \in \mathrm{C}(P) .
$$

Suppose that $T[X] \subseteq Y$. If $Y$ is a Riesz subspace of $\mathrm{C}(Q)$, then $T: X \rightarrow Y$ has a modulus, and, for every $x \in X$, we have

$$
(|T| x)(q)=|w(q)| x(\alpha(q)), \quad q \in Q
$$

Proof Denote $(S x)(q):=|w(q)| x(\alpha(q))$ for $q \in Q$ and $x \in \mathrm{C}(P)$. First, we show that $S[X] \subseteq Y$. Indeed, for $x \in X, x \geq 0$, we have

$$
(S x)(q)=|w(q)| x(\alpha(q))=|w(q) x(\alpha(q))|=|(T x)(q)|
$$

for every $q \in Q$. As $T[X] \subseteq Y$ and $Y$ is a Riesz subspace of $\mathrm{C}(Q)$, we get $|T x| \in Y$ and, hence, $S x \in Y$. As $X$ is directed, we obtain $S[X] \subseteq Y$, and $S: X \rightarrow Y$ is a positive linear map.

Next, we show that $S$ is the modulus of $T$. Indeed, for every $x \in X, x \geq 0$, we have $S x \geq \pm T x$, hence $S \geq \pm T$. If $R: X \rightarrow Y$ is a linear map such that $R \geq \pm T$, then for every $x \in X, x \geq 0$, we have $R x \geq T x$ and $R x \geq-T x$. For every $q \in Q$, we then obtain

$$
(R x)(q) \geq|(T x)(q)|=|w(q) x(\alpha(q))|=|w(q)| x(\alpha(q))=(S x)(q),
$$

hence $R \geq S$. Thus, $S$ is the supremum of $\{T,-T\}$.

Remark 61 If $Y$ is a pre-Riesz space which is order dense in $\mathrm{C}(Q)$, then $Y^{\rho}$ is a Riesz subspace of $\mathrm{C}(Q)$, and $Y \subseteq Y^{\rho}$. In this case, $T: X \rightarrow Y$ has a modulus in the regular operators from $X$ to $Y^{\rho}$.

It is an open question under which conditions the difference of two Riesz* homomorphisms has a modulus. In view of Corollary 36 , this leads to the question under 
which conditions two Riesz* homomorphisms are disjoint. We give a partial answer in the setting of Proposition 60. Let $P$ be a nonempty compact Hausdorff space and let $X \subseteq \mathrm{C}(P)$ be a linear subspace. We will use the following property of $X$, which we call strongly pointwise pervasive:

(SPP) $\forall z \in \mathrm{C}(P)_{+} \forall p \in P: z(p)=\sup \{x(p) ; x \in X, 0 \leq x \leq z\}$.

Lemma 62 If $X$ satisfies (SPP), then $X$ is pointwise pervasive.

Proof Let $p \in P$ and let $U$ be a neighbourhood of $p$. Take $U_{0} \subseteq P$ open with $p \in U_{0} \subseteq U$. By Urysohn's lemma, there exists a $z \in \mathrm{C}(P)$ with $z(p)=1$ and $z=0$ on $P \backslash \bar{U}_{0}$. According to (SPP), there exists $x \in X$ with $0 \leq x \leq z$ and $x(p) \geq \frac{1}{2}$. Then $x=0$ on $P \backslash U_{0} \supseteq P \backslash U$. Thus, $X$ is pointwise pervasive.

As a consequence of Lemma 62 and Proposition 50, we obtain that (SPP) implies (SD).

Theorem 63 Let $P$ and $Q$ be nonempty compact Hausdorff spaces and let $X \subseteq \mathrm{C}(P)$ and $Y \subseteq \mathrm{C}(Q)$ be linear subspaces, where $X$ is directed and satisfies (SPP). Let $T_{1}, T_{2}: X \rightarrow Y$ be given by

$$
\left(T_{i} x\right)(q):=w_{i}(q) x\left(\alpha_{i}(q)\right), \quad q \in Q, i \in\{1,2\}
$$

where $\alpha_{1}, \alpha_{2}: Q \rightarrow P$ and $w_{1}, w_{2}: Q \rightarrow \mathbb{R}_{+}$. Consider the condition

$\left\{q \in Q ; w_{1}(q)=0\right.$ or $w_{2}(q)=0$ or $\left.\alpha_{1}(q) \neq \alpha_{2}(q)\right\}$ is dense in $Q$.

(i) If (20) holds, then $T_{1} \perp T_{2}$ in $\mathrm{L}(X, Y)$. Hence, $T_{1}-T_{2}$ has a modulus which equals $T_{1}+T_{2}$.

(ii) If, in addition, $Y$ is pervasive and, for every $y_{1}, y_{2} \in Y$, we have $y_{1} \cdot y_{2} \in Y$, then (20) holds if and only if $T_{1} \perp T_{2}$ in $\mathrm{L}(X, Y)$.

Proof (i) As $T_{1}$ and $T_{2}$ are positive, showing that $T_{1} \perp T_{2}$ in $\mathrm{L}(X, Y)$ comes down to showing that, for every $U \in \mathrm{L}(X, Y)$, we have

$$
\left(U \geq T_{1}-T_{2} \text { and } U \geq T_{2}-T_{1}\right) \Rightarrow U \geq T_{1}+T_{2}
$$

Let $U \in \mathrm{L}(X, Y)$ be such that $U \geq T_{1}-T_{2}$ and $U \geq T_{2}-T_{1}$. Fix $x \in X$ with $x \geq 0$ and $q \in Q$. If $w_{1}(q)=0$, then $\left(T_{1} x\right)(q)=0$, hence $(U x)(q) \geq\left(T_{2} x\right)(q)=$ $\left(\left(T_{1}+T_{2}\right) x\right)(q)$. Similarly, if $w_{2}(q)=0$, then $(U x)(q) \geq\left(\left(T_{1}+T_{2}\right) x\right)(q)$.

Now assume $w_{1}(q) \neq 0, w_{2}(q) \neq 0$, and $\alpha_{1}(q) \neq \alpha_{2}(q)$. As the condition (SD) follows from (SPP) for $X$, there exist $a_{1}, a_{2} \in X$ with $a_{1}, a_{2} \geq 0$ such that $a_{1} \perp a_{2}$ and $a_{i}\left(\alpha_{i}(q)\right)=x\left(\alpha_{i}(q)\right)$ for $i \in\{1,2\}, a_{1}\left(\alpha_{2}(q)\right)=0$ and $a_{2}\left(\alpha_{1}(q)\right)=0$. Put $z_{1}:=x \wedge a_{1}$ and $z_{2}:=x \wedge a_{2}$. Let $\varepsilon>0$. By (SPP), there exist $y_{1}, y_{2} \in X$ with $0 \leq y_{1} \leq z_{1}$ and $0 \leq y_{2} \leq z_{2}$ such that

$$
y_{i}\left(\alpha_{i}(q)\right) \geq z_{i}\left(\alpha_{i}(q)\right)-\varepsilon, \quad i \in\{1,2\} .
$$


Then $0 \leq y_{1} \wedge y_{2} \leq a_{1} \wedge a_{2}=0$, therefore $y_{1} \perp y_{2}$. Hence,

$$
0 \leq y_{1}+y_{2}=y_{1} \vee y_{2} \leq z_{1} \vee z_{2} \leq x
$$

Furthermore,

$$
\begin{aligned}
\left(U y_{1}\right)(q) & \geq\left(\left(T_{1}-T_{2}\right) y_{1}\right)(q)=w_{1}(q) y_{1}\left(\alpha_{1}(q)\right)-w_{2}(q) y_{1}\left(\alpha_{2}(q)\right) \\
& =w_{1}(q) y_{1}\left(\alpha_{1}(q)\right) \geq w_{1}(q)\left(z_{1}\left(\alpha_{1}(q)\right)-\varepsilon\right) \\
& =w_{1}(q)\left(x\left(\alpha_{1}(q)\right)-\varepsilon\right)
\end{aligned}
$$

Similarly,

$$
\begin{aligned}
\left(U y_{2}\right)(q) & \geq\left(\left(T_{2}-T_{1}\right) y_{2}\right)(q)=w_{2}(q) y_{2}\left(\alpha_{2}(q)\right)-w_{1}(q) y_{2}\left(\alpha_{1}(q)\right) \\
& \geq w_{2}(q)\left(x\left(\alpha_{2}(q)\right)-\varepsilon\right) .
\end{aligned}
$$

Thus,

$$
\begin{aligned}
(U x)(q) & \geq\left(U\left(y_{1}+y_{2}\right)\right)(q) \\
& \geq w_{1}(q) x\left(\alpha_{1}(q)\right)+w_{2}(q) x\left(\alpha_{2}(q)\right)-\left(w_{1}(q)+w_{2}(q)\right) \varepsilon \\
& =\left(\left(T_{1}+T_{2}\right) x\right)(q)-\left(w_{1}(q)+w_{2}(q)\right) \varepsilon .
\end{aligned}
$$

Hence $(U x)(q) \geq\left(\left(T_{1}+T_{2}\right) x\right)(q)$. Because of (20), it follows by continuity that $U x \geq\left(T_{1}+T_{2}\right) x$. Hence, $U \geq T_{1}+T_{2}$ and, therefore,

$$
\left\{T_{1}-T_{2}, T_{2}-T_{1}\right\}^{\mathrm{u}}=\left\{T_{1}+T_{2},-T_{1}-T_{2}\right\}^{\mathrm{u}}
$$

in $\mathrm{L}(X, Y)$, which means that $T_{1} \perp T_{2}$. By Corollary 36, the operator $T_{1}-T_{2}$ has a modulus which equals $T_{1}+T_{2}$.

(ii) Suppose (20) does not hold. Then there exists a nonempty open set $V \subseteq Q$ such that $w_{1} \neq 0, w_{2} \neq 0$ and $\alpha_{1}=\alpha_{2}$ on $V$. By Urysohn's Lemma, there exists a function $v_{0} \in \mathrm{C}(Q)$ with $0 \leq v_{0} \leq 1$ such that $\operatorname{coz}\left(v_{0}\right) \subseteq V$ and $v_{0} \neq 0$. By the pervasiveness of $Y$, there is $v \in Y_{+} \backslash\{0\}$ with $v(q) \leq \min \left\{1, \frac{w_{2}(q)}{w_{1}(q)}\right\} v_{0}(q)$ for every $q \in Q$. Choose $q_{0} \in V$ such that $v\left(q_{0}\right)>0$. For every $x \in X, q \in Q$, define

$$
(U x)(q):=\left(\left(T_{1}+T_{2}\right) x\right)(q)-v(q) T_{1} x(q) .
$$

Since $v, T_{1} x \in Y$ and $Y$ is closed under multiplication, we have $U \in \mathrm{L}(X, Y)$. We show that $U \geq \pm\left(T_{1}-T_{2}\right)$. Indeed, let $x \in X, x \geq 0$, and let $q \in Q$. If $q \notin V$, then

$$
\left( \pm\left(T_{1}-T_{2}\right) x\right)(q) \leq\left(\left(T_{1}+T_{2}\right) x\right)(q)=(U x)(q) .
$$


If $q \in V$, then, as $w_{2}(q)-v(q) w_{1}(q) \geq 0$, we have

$$
\begin{aligned}
(U x)(q) & =w_{1}(q) x\left(\alpha_{1}(q)\right)+w_{2}(q) x\left(\alpha_{2}(q)\right)-v(q) w_{1}(q) x\left(\alpha_{1}(q)\right) \\
& =\left(w_{1}(q)+w_{2}(q)-v(q) w_{1}(q)\right) x\left(\alpha_{1}(q)\right) \\
& \geq w_{1}(q) x\left(\alpha_{1}(q)\right)=\left(T_{1} x\right)(q) \\
& \geq\left(\left(T_{1}-T_{2}\right) x\right)(q),
\end{aligned}
$$

and, similarly, as $v(q) w_{1}(q) \leq w_{1}(q)$,

$$
\begin{aligned}
(U x)(q) & =\left(w_{1}(q)+w_{2}(q)-v(q) w_{1}(q)\right) x\left(\alpha_{1}(q)\right) \\
& \geq w_{2}(q) x\left(\alpha_{1}(q)\right)=w_{2}(q) x\left(\alpha_{2}(q)\right)=\left(T_{2} x\right)(q) \\
& \geq\left(\left(T_{2}-T_{1}\right) x\right)(q) .
\end{aligned}
$$

Thus, $U \geq \pm\left(T_{1}-T_{2}\right)$. Next, we show $U \nsupseteq T_{1}+T_{2}$. Indeed, by (SPP), the space $X$ is pointwise pervasive, hence there exists $x \in X, x \geq 0$, with $x\left(\alpha_{1}\left(q_{0}\right)\right)>0$. Then

$$
\begin{aligned}
(U x)\left(q_{0}\right) & =\left(w_{1}\left(q_{0}\right)+w_{2}\left(q_{0}\right)-v\left(q_{0}\right) w\left(q_{0}\right)\right) x\left(\alpha_{1}\left(q_{0}\right)\right) \\
& <\left(w_{1}\left(q_{0}\right)+w_{2}\left(q_{0}\right)\right) x\left(\alpha_{1}\left(q_{0}\right)\right) \\
& =\left(\left(T_{1}+T_{2}\right) x\right)\left(q_{0}\right) .
\end{aligned}
$$

Thus, $U \in\left\{T_{1}-T_{2}, T_{2}-T_{1}\right\}^{\mathrm{u}}$, but $U \notin\left\{T_{1}+T_{2},-T_{1}-T_{2}\right\}^{\mathrm{u}}$, which means that $T_{1}$ and $T_{2}$ are not disjoint in $\mathrm{L}(X, Y)$.

Remark 64 Note that (20) is satisfied whenever the graphs

$$
\left\{\left(q, \alpha_{1}(q)\right) ; q \in Q\right\} \text { and }\left\{\left(q, \alpha_{2}(q)\right) ; q \in Q\right\}
$$

are disjoint sets. For the proof of Theorem 63(ii) that (20) is a necessary condition for $T_{1} \perp T_{2}$, we use that $X$ is pointwise pervasive. It is open whether (SPP) is strictly stronger than being pointwise pervasive.

Remark 65 If $w_{1}, w_{2}, \alpha_{1}, \alpha_{2}$ in Theorem 63 are continuous, the statement there is about the modulus of differences of Riesz* homomorphisms by Theorem 12. This can be specified further.

In [12], a subspace $X$ of $\mathrm{C}(P)$ is called pointwise order dense if, for every $z \in \mathrm{C}(P)$ and $p \in P$, we have $z(p)=\inf \{x(p) ; x \in X, x \geq z\}$. If $X$ is a majorizing subspace of $\mathrm{C}(P)$ that satisfies (SPP), then $X$ is pointwise order dense. Indeed, let $z \in \mathrm{C}(P)$, $p \in P$. As $X$ is majorizing, there is $v \in X, v \geq z$. By (SPP), we have

$$
\begin{aligned}
(v-z)(p) & =\sup \{x(p) ; x \in X, 0 \leq x \leq v-z\}, \text { hence } \\
(z-v)(p) & =\inf \{-x(p) ; x \in X, 0 \leq x \leq v-z\}, \text { consequently } \\
z(p) & =\inf \{(v-x)(p) ; x \in X, 0 \leq x \leq v-z\} \\
& =\inf \{w(p) ; w \in X, z \leq w\} .
\end{aligned}
$$


In [12, Theorem 3.4], the following is shown: if $X$ is pointwise order dense in $\mathrm{C}(P)$ and $Y$ is order dense in $\mathrm{C}(Q)$, then every Riesz* homomorphism is a Riesz homomorphism. Therefore, if, in addition to the assumptions in Theorem 63 and the continuity of $w_{1}, w_{2}, \alpha_{1}, \alpha_{2}, X$ is majorizing and $Y$ is order dense, the statement there is for differences of Riesz homomorphisms.

Let $P$ be a nonempty compact Hausdorff space, $p \in P$, and $X$ a linear subspace of $\mathrm{C}(P)$. We denote $\varphi_{p}: X \rightarrow \mathbb{R}, \varphi_{p}(x):=x(p)$.

Corollary 66 Let $P$ be a nonempty compact Hausdorff space and let $X \subseteq \mathrm{C}(P)$ be a linear directed subspace which satisfies $(S P P)$. Then, for every $p_{1}, p_{2} \in P$, we have $p_{1} \neq p_{2}$ if and only if $\varphi_{p_{1}} \perp \varphi_{p_{2}}$.

Proof Let $p_{1}, p_{2} \in P, p_{1} \neq p_{2}$. We view $\varphi_{p_{i}}(i \in\{1,2\})$ as a map from $X$ to $\mathrm{C}(Q)$, where the set $Q$ consists of one point $q$, and, for every $x \in X$, we interpret the real number $\varphi_{p_{i}}(x)$ as the continuous function $q \mapsto \varphi_{p_{i}}(x)(i \in\{1,2\})$. Define $\alpha_{i}: Q \rightarrow P$ by $\alpha_{i}(q)=p_{i}(i \in\{1,2\})$. Hence, we have, for $x \in X$, that $\left(\varphi_{p_{i}}(x)\right)(q)=$ $x\left(p_{i}\right)=x\left(\alpha_{i}(q)\right)$ for every $q \in Q$. As the graphs of $\alpha_{1}$ and $\alpha_{2}$ are disjoint, Theorem 63 yields that $\varphi_{p_{1}} \perp \varphi_{p_{2}}$.

If $p_{1}=p_{2}$, then obviously $\varphi_{p_{1}}=\varphi_{p_{2}}$, hence $\varphi_{p_{1}} \not \perp \varphi_{p_{2}}$.

Corollary 67 Let $P$ and $Q$ be nonempty compact Hausdorff spaces and let $X \subseteq \mathrm{C}(P)$ and $Y \subseteq \mathrm{C}(Q)$ be linear subspaces, where $X$ is directed and satisfies $(S P P)$. Let $T_{1}, T_{2}: X \rightarrow Y$ be given by

$$
\left(T_{i} x\right)(q)=w_{i}(q) x\left(\alpha_{i}(q)\right), \quad q \in Q, i \in\{1,2\},
$$

where $\alpha_{1}, \alpha_{2}: Q \rightarrow P$ and $w_{1}, w_{2}: Q \rightarrow \mathbb{R}_{+}$. The set

$$
\left\{q \in Q ; \varphi_{q} \circ T_{1} \perp \varphi_{q} \circ T_{2}\right\}
$$

is dense in $Q$ if and only if $T_{1} \perp T_{2}$ in $\mathrm{L}(X, \mathrm{C}(Q))$.

Proof Fix $q \in Q$. For every $i \in\{1,2\}$ and $x \in X$ we have that

$$
\left(\varphi_{q} \circ T_{i}\right)(x)=w_{i}(q) x\left(\alpha_{i}(q)\right)
$$

Hence, $\left(\varphi_{q} \circ T_{i}\right)=w_{i}(q) \varphi_{\alpha_{i}(q)}$. We have

$$
\begin{aligned}
\varphi_{q} \circ T_{1} \perp \varphi_{q} \circ T_{2} & \Longleftrightarrow w_{1}(q) \varphi_{\alpha_{1}(q)} \perp w_{2}(q) \varphi_{\alpha_{2}(q)} \\
& \Longleftrightarrow w_{1}(q)=0 \text { or } w_{2}(q)=0 \text { or } \alpha_{1}(q) \neq \alpha_{2}(q),
\end{aligned}
$$

by Corollary 66. Now we apply Theorem 63, which completes the proof.

Remark 68 If $Y$ is a subspace of $\mathrm{C}(Q)$, then $\mathrm{L}(X, Y)$ is a subspace of $\mathrm{L}(X, \mathrm{C}(Q))$. Hence, for $T_{1}, T_{2} \in \mathrm{L}(X, Y)$, we have

$$
T_{1} \perp T_{2} \text { in } \mathrm{L}(X, \mathrm{C}(Q)) \quad \Longrightarrow \quad T_{1} \perp T_{2} \text { in } \mathrm{L}(X, Y) \text {. }
$$


In the subsequent corollary, the space $X$ is a pre-Riesz space. We use Theorem 5 to obtain a unique extension for Riesz* homomorphisms on $X$.

Corollary 69 Let $P$ and $Q$ be nonempty compact Hausdorff spaces and let $X \subseteq \mathrm{C}(P)$ be a linear subspace, where $X$ is directed and satisfies $(S P P)$. Let $T_{1}, T_{2}: X \rightarrow \mathrm{C}(Q)$ be Riesz* homomorphisms and let $S_{1}, S_{2}: X^{\rho} \rightarrow \mathrm{C}(P)$ be the Riesz homomorphisms extending $T_{1}$ and $T_{2}$, respectively. Then

$$
T_{1} \perp T_{2} \text { in } \mathrm{L}(X, \mathrm{C}(Q)) \Longleftrightarrow S_{1} \perp S_{2} \text { in } \mathrm{L}\left(X^{\rho}, \mathrm{C}(Q)\right) \text {. }
$$

Proof According to Theorem 12, there exist $w_{1}, w_{2}: Q \rightarrow \mathbb{R}_{+}$and $\alpha_{1}, \alpha_{2}: Q \rightarrow P$ such that, for every $i \in\{1,2\}, x \in X$, and $q \in Q$, we have

$$
\left(T_{i} x\right)(q)=w_{i}(q) x\left(\alpha_{i}(q)\right)
$$

As $x \mapsto\left(q \mapsto w_{i}(q) x\left(\alpha_{i}(q)\right)\right)$ is a Riesz homomorphism from $X^{\rho}$ to $\mathrm{C}(Q)$ extending $T_{i}$, we obtain

$$
\left(S_{i} x\right)(q)=w_{i}(q) x\left(\alpha_{i}(q)\right)
$$

for every $i \in\{1,2\}, x \in X^{\rho}$, and $q \in Q$, due to the uniqueness of the extension. Now Theorem 63 completes the proof.

Theorem 63 considers operators that have a representation as weighted composition operators. These operators are Riesz* homomorphisms. A natural next step would be to investigate disjointness of two Riesz* homomorphisms between arbitrary pre-Riesz spaces $X$ and $Y$. Corollary 69 raises the question under which conditions on $X$ and $Y$ we obtain that two Riesz* homomorphisms from $X$ to $Y$ are disjoint if and only if their unique extensions from $X^{\rho}$ to $Y^{\rho}$ are disjoint.

Funding Open Access funding enabled and organized by Projekt DEAL.

Open Access This article is licensed under a Creative Commons Attribution 4.0 International License, which permits use, sharing, adaptation, distribution and reproduction in any medium or format, as long as you give appropriate credit to the original author(s) and the source, provide a link to the Creative Commons licence, and indicate if changes were made. The images or other third party material in this article are included in the article's Creative Commons licence, unless indicated otherwise in a credit line to the material. If material is not included in the article's Creative Commons licence and your intended use is not permitted by statutory regulation or exceeds the permitted use, you will need to obtain permission directly from the copyright holder. To view a copy of this licence, visit http://creativecommons.org/licenses/by/4.0/.

\section{References}

1. Abramovich, Y.A., Wickstead, A.W.: Regular operators from and into small Riesz spaces. Indag. Math. N.S. 2, 257-274 (1991)

2. Aliprantis, C.D., Burkinshaw, O.: Positive Operators. Academic Press Inc., London (1985)

3. Aliprantis, C.D., Tourky, R.: Cones and Duality. Graduate Studies in Mathematics, vol. 84. American Mathematical Society, Rhode Island (2007) 
4. Aliprantis, C.D., Tourky, R., Yannelis, N.C.: The Riesz-Kantorovich formula and general equilibrium theory. J. Math. Econ. 34, 55-76 (2000)

5. Buskes, G., van Rooij, A.C.M.: The vector lattice cover of certain partially ordered groups. J. Austral. Math. Soc. (Series A) 54, 352-367 (1993)

6. de Jeu, M., Jiang, X.: Riesz representation theorems for positive algebra homomorphisms, Preprint

7. van Gaans, O., Kalauch, A.: Bands in pervasive pre-Riesz spaces. Oper. Matrices 2(2), 177-191 (2008)

8. Kalauch, A., Lemmens, B., van Gaans, O.: Riesz completions, functional representations, and antilattices. Positivity 18(1), 201-218 (2014)

9. Kalauch, A., van Gaans, O.: Pre-Riesz Spaces. Walter de Gruyter GmbH, Berlin/Boston (2019)

10. van Haandel, M.: Completions in Riesz space theory, Ph.D. thesis, University of Nijmegen (1993)

11. Hayes, A.: Indecomposable positive additive functionals. J. London Math. Soc. 41, 318-322 (1966)

12. van Imhoff, H.: Riesz* homomorphisms on pre-Riesz spaces consisting of continuous functions. Positivity 22(2), 425-447 (2018)

13. van Imhoff, H.: Order isomorphisms, order antimorphisms and their interplay with Jordan algebra structures. Ph.D. thesis, Universiteit Leiden (2019)

14. Kalauch, A.., van Gaans, O.., Zhang, F..: Disjointness preserving operators on normed pre-Riesz spaces: extensions and inverses. Positivity 24(2), 481-504 (2020)

15. Megginson, R.E.: An Introduction to Banach Space Theory. Graduate Texts in Mathematics, vol. 183. Springer, New York (1998)

16. Zaanen, A.C.: Introduction to Operator Theory in Riesz Spaces. Springer, Berlin (1997)

Publisher's Note Springer Nature remains neutral with regard to jurisdictional claims in published maps and institutional affiliations. 